\title{
EchoGéo
}

\section{"Retour" et "reconquête" de la péninsule historique : discours et usages distinctifs autour du patrimoine de Fener et Çarşamba}

\section{Brian Chauvel}

\section{OpenEdition}

Journals

Édition électronique

URL : https://journals.openedition.org/echogeo/12463

DOI : $10.4000 /$ echogeo. 12463

ISSN : 1963-1197

Éditeur

Pôle de recherche pour l'organisation et la diffusion de l'information géographique (CNRS UMR 8586)

Référence électronique

Brian Chauvel, " "Retour" et "reconquête" de la péninsule historique : discours et usages distinctifs autour du patrimoine de Fener et Çarşamba », EchoGéo [En ligne], 16 | 2011, mis en ligne le 04 juillet 2011, consulté le 25 août 2021. URL : http://journals.openedition.org/echogeo/12463 ; DOI : https:// doi.org/10.4000/echogeo.12463

Ce document a été généré automatiquement le 25 août 2021.

EchoGéo est mis à disposition selon les termes de la licence Creative Commons Attribution - Pas d'Utilisation Commerciale - Pas de Modification 4.0 International (CC BY-NC-ND) 


\title{
"Retour" et "reconquête" de la péninsule historique : discours et usages distinctifs autour $\mathrm{du}$ patrimoine de Fener et Çarşamba
}

\author{
Brian Chauvel
}

\section{Introduction : retours vers les quartiers anciens et reconfiguration de l'espace social}

1 Le 8 mars 1998, Oktay Ekinci, ancien président de la chambre des architectes (Mimarlar Odasl) et défenseur du patrimoine naturel et historique, affirmait, dans l'édition anglophone Hürriyet Daily News, la nécessité que «les habitants vivant dans les zones forestières hors de la ville retournent dans les centres-villes et partagent leurs vies avec les autres $»^{1}$. Dans son propos, le thème du "retour» apparaissait comme une réponse à l'éclatement socio-spatial d'Istanbul et particulièrement à son développement périphérique, clivé entre ces cités privées et des zones d'habitat spontané. Arguant que les habitants légitimes de la ville sont ceux qui n'auraient jamais quitté ces quartiers anciens, il évoquait aussi des "propriétés mystiques " laissant augurer une possible communion avec des classes populaires, depuis l'espace retrouvé et bientôt restauré de la péninsule historique et des quartiers de Beyoğlu. La préservation du patrimoine stambouliote peut ainsi apparaître comme la tête de pont d'un processus de changement social qui passerait par une modification du rapport à certains espaces et à leurs habitants; appelant à l'investissement de ces lieux par des classes plus aisées, le thème du retour dissimulerait une gentrification.

Derrière un processus interprétable à une échelle globale, se joue, autour de secteurs classés au patrimoine mondial de l'humanité depuis 1985, une scène qui met en lumière des acteurs et des conceptions différentes voire antagoniques du patrimoine. Si ces acteurs multiples s'inscrivent dans la lignée d'institutions nationales (telle la 
Commission pour la conservation des œuvres anciennes créée en 1924) et internationales (la Turquie est signataire de la convention concernant la protection du patrimoine mondial, culturel et naturel adoptée par la conférence générale de l'UNESCO - novembre 1972), il est toutefois « très réducteur » de lier ce phénomène à la « prétendue occidentalisation de la société turque ». Certes, la question du patrimoine à Istanbul est portée par une « société civile » et des figures telles Oktay Ekinci (Gürsoy \& Hüküm, 2006) devenues plus audibles depuis la conférence Habitat II (juin 1997). Mais, « dès le début des années 1940, on peut identifier des initiatives tendant à développer une conscience patrimoniale dans la perspective de fêter le $500^{\mathrm{e}}$ anniversaire de la conquête d'Istanbul» (Pérouse, 2010: 257), initiatives qui trouvent une audience croissante depuis l'arrivée du Refah à la tête de la municipalité d'Istanbul en 1994.

3 Ainsi, le thème du "retour» fait face à son double, le "paradigme de la conquête » (Pérouse, 2003) étant porté par un discours politique qui le lie à un projet de refonte du lien social à travers diverses références, souvent idéologiques, à l'Islam. Le Refah, parti " du bien être », et son jeune candidat Recep Tayyip Erdoğan, après avoir fait campagne sur le thème de la conquête d'Istanbul pour prendre la tête de cette Grande Municipalité en 1994, ont en effet accordé une importance toute particulière à la péninsule historique, à un arrondissement dont le nom a une forte valeur symbolique. Fatih («le conquérant » en turc) étant une référence directe à l'ancrage de l'Empire ottoman dans un centre urbain dont les éléments symboliques majeurs sont réinterprétés à la lumière d'un changement de souveraineté (la perpétuation du statut de capitale impériale s'accompagnant d'un complexe agencement de ruptures et de continuités avec les références byzantines - Yerasimos, 2000), ses monuments, mosquées et mausolées relèvent d'un héritage emblématique, sinon polémique ${ }^{2}$.

La " conquête » étant conçue comme un retour conservateur à la fois vers le peuple, vers des racines islamiques et vers la ville historique, j'émets ici l'hypothèse que la mise en valeur du patrimoine bâti, sa conservation, participerait aussi à la légitimation ${ }^{3}$ de l'ascension sociale et des trajectoires résidentielles de populations originaires des provinces anatoliennes, de nouvelles classes aisées qui se distingueraient des anciennes élites stambouliotes par un usage « moderne » des références à l'Islam (Göle, 2003). Les processus de patrimonialisation œuvrent en effet à une reconfiguration de l'espace public qui peut être rapprochée de celle engendrée par le port du voile; se fixant dans le bâti, ce discours réinterprète des éléments tenus pour traditionnels, les esthétise et les érige en symboles d'une modernité non occidentale (Göle, 2000).

Conférant un caractère durable à ces marqueurs, le discours conservateur voile cependant aussi les réinterprétations et retraductions de l'espace auxquelles il procède. L'exemple de la rénovation et de l'ampleur des destructions en cours dans le quartier de Sülemaniye, pourtant classé au patrimoine mondial, montre en effet que derrière les façades en bois-plaqué d'un bâti présenté comme l'archétype d'un habitat ottoman se joue une toute autre scène; l'apparente permanence et continuité d'un patrimoine "vernaculaire $»^{4}$ qui sera photographié par les touristes et vendu à des propriétaires fortunés dissimule des processus d'éviction forcée de familles et de célibataires qui trouvaient dans ce quartier des possibilités de logement à bas coût proche des ateliers et boutiques d'Eminönü (Renou, 2010 ; şeni, 2010). La question du patrimoine, quelle que soit sa charge symbolique, n'échappe ainsi absolument pas aux logiques économiques; la municipalité cherche à déplacer l'ensemble des activités de 
production subsistant sur la péninsule historique, pensant intégrer ces anciens quartiers au projet « Istanbul ville musée » (2004) qui vise à développer le tourisme.

Le thème du retour vers les vieux quartiers d'Istanbul semble ainsi porteur de distinctions sociales qui se cristallisent autour du patrimoine de la péninsule historique. D'une part, au regard des logiques de champ autour de cette question, on peut en effet supposer que ce thème nourrit une opposition tendancielle et une concurrence entre des classes aisées 'occidentalisées' appelées à revenir vers leurs anciens quartiers et des classes montantes anatoliennes incitées à retrouver les sources d'un héritage ottoman qui serait turc et islamique. Mais cette hypothèse doit être relativisée dans la mesure où, avant de quantifier la part d'habitants des 'site' périphériques effectivement originaires des anciens quartiers centraux et celle des 'anatoliens', il faudrait d'abord faire la généalogie de cette distinction et surtout en interroger la pertinence scientifique. D'autre part, qu'il s'agisse d'un « retour » vers ces espaces ou d'une « conquête», ce mouvement présuppose une distance initiale envers des habitants qui en seraient pourtant soit «les propriétaires légitimes » soit les " héritiers». Or, des évictions en témoignent, cette distance initiale se translate en éloignement (notamment dans des logements TOKI construits en périphérie).

7 Les quartiers de Fener et de Çarşamba semblent dès lors intéressants pour interroger un processus complexe dont on peut observer certaines traductions dans la mise en scène des espaces patrimonialisés et de leurs alentours. Voisins, ils autorisent une comparaison qui permet de penser les interactions entre les discours précités, des héritages a priori distincts et des pratiques de consommation distinctives. La partie basse de ce secteur, communément appelée Fener, abrite le Patriarcat Rum et a fait l'objet d'une réhabilitation menée avec la mairie de l'arrondissement de Fatih dans le cadre d'un programme européen. En hauteur, le quartier de Çarşamba, dominé par la mosquée de Yavuz Sultan Selim, est l'objet de mises en valeur plus ponctuelles, d'initiatives privées ou liées à des fondations pieuses. Les traverser permet de tester, sans les forcer, quelques hypothèses (émises au préalable).

\section{Le patrimoine bâti de Fener et Çarşamba : frontières et continuités}

8 Issu du maillage administratif de 2009, le quartier (mahalle) de Balat, regroupe plusieurs aires de voisinage (semt), dont Fener et Çarşamba. Ces espaces vécus «en termes de seuils, de contacts, d'articulations ou de coupures avec leur environnement urbain » (Teboul, 2002) sont appréhendables depuis leurs frontières, dans leurs interrelations et confrontations. Fener est habituellement étudié en lien avec Balat. Le projet de réhabilitation " des quartiers de Fener et de Balat » (Stoquart, 1997), a en effet délimité un périmètre qui retient l'attention des études portant sur les projets urbains, de « rénovation », « renouvellement » ou « réhabilitation». Or si, plutôt que d'étudier le cas de Fener en lien avec Balat, on porte le regard vers les hauteurs de Çarşamba, apparaît un contraste frappant qui mérite d'être examiné à la lumière du contexte contemporain de "reconquête" de la péninsule historique. 


\section{De Fener à Çarşamba, un seuil marqué par le dénivelé et renforcé par l'architecture.}

La topographie est d'un premier secours pour percevoir les frontières de Fener et de Çarşamba. Çarşamba est situé en haut d'une colline de 50 mètres (carte 1), surplombée par la mosquée Sultan Selim et reliée à la grande mosquée de Fatih et sa medresse par une ligne de crête. Fener se trouve en contrebas, quelques mètres à peine au dessus des eaux de la Corne d'or, adossé à un coteau qui s'ouvre sur Balat et se prolonge jusqu'Ayvansaray et la muraille terrestre. Les pentes, qui ont contenu les incendies ravageurs $\mathrm{du} \mathrm{XIX}^{\mathrm{e}}$ siècle, délimitent une zone au bâti relativement homogène. Protégées par des murs de soutènement, d'anciennes maisons en bois restèrent accrochées aux coteaux, tandis que l'intérieur fut radicalement transformé après le grand incendie de 1866. Le tissu urbain situé en dessous fut alors réorganisé selon un plan orthogonal alignant des maisons en bande à encorbellement de deux à trois étages, ce qui lui donne des « caractéristiques architecturales et urbaines distinctives ». Construites en dur, ces maisons ont en effet traversé le $\mathrm{XX}^{\mathrm{e}}$ siècle et, classées au patrimoine mondial en 1985 , ont été l'objet du programme «de réhabilitation des quartiers de Fener et de Balat», mené entre 1997 et 2008 par la Mairie d'arrondissement de Fatih et l'Union européenne (carte 2).

\section{Carte 1 - Dénivelé entre Fener et Çarşamba}

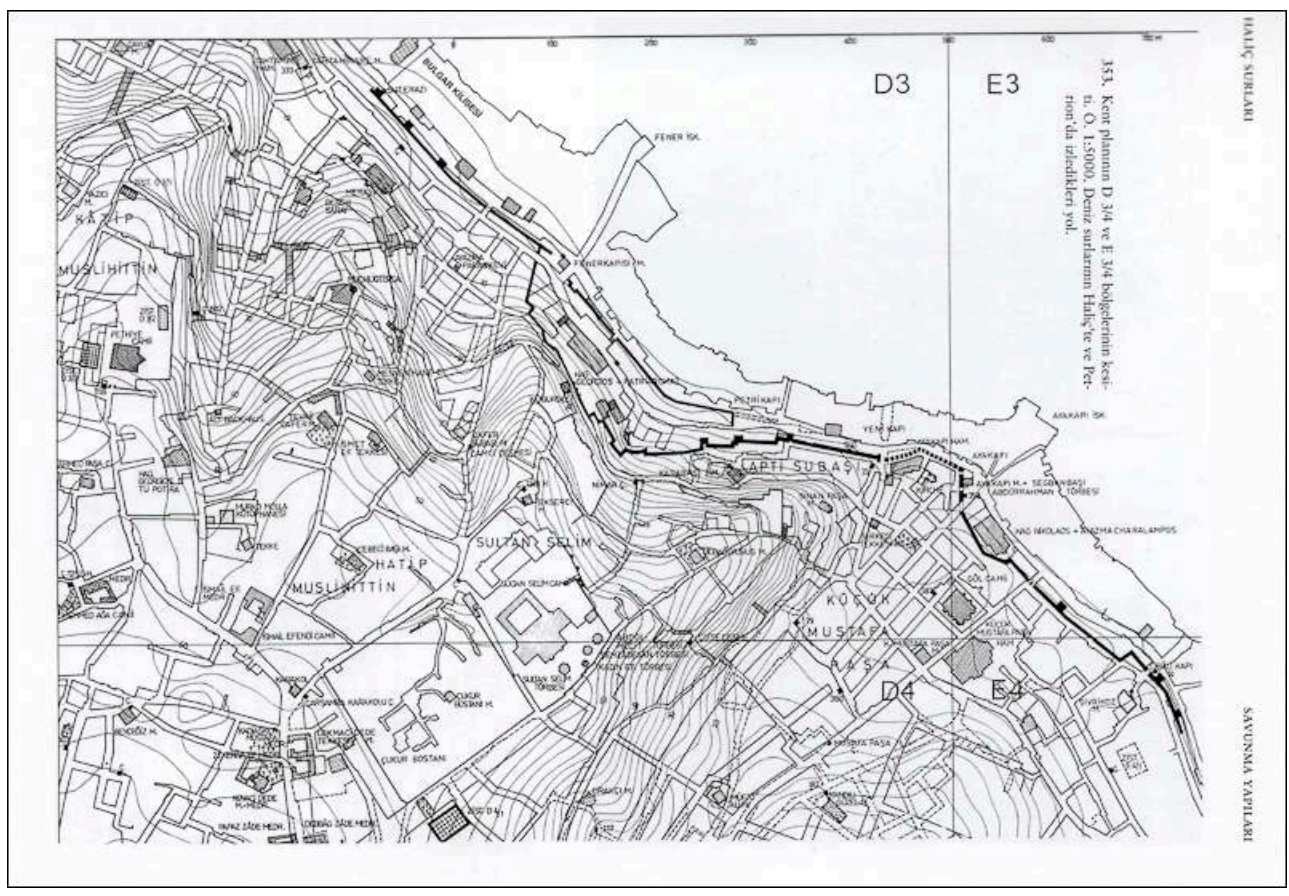

Source : Müller-Wiener Wolfgang, Istanbul'un Tarihsel Topografyasi, Yapi Kredi Kültür Merkez, Istanbul, 2001 
Carte 2 - Délimitation du projet de réhabilitation des quartiers de Balat et de Fener

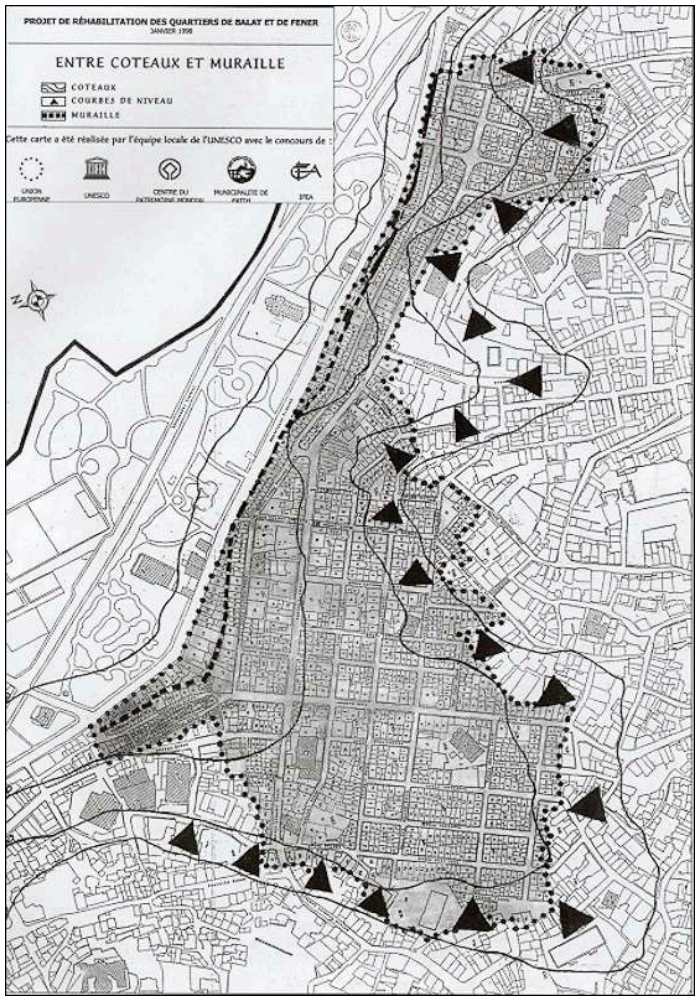

Source : « Entre coteaux et muraille », Projet de Réhabilitation des quartiers de Balat et de Fener, Rapport dirigé par Rémi Stoquart, Union Européenne, Centre du patrimoine mondial de I'UNESCO, Municipalité de Fatih, IFEA, 1998. 
Illustration 1 - Maisons à encorbellement en bande dans la rue Baki Dede, Fener

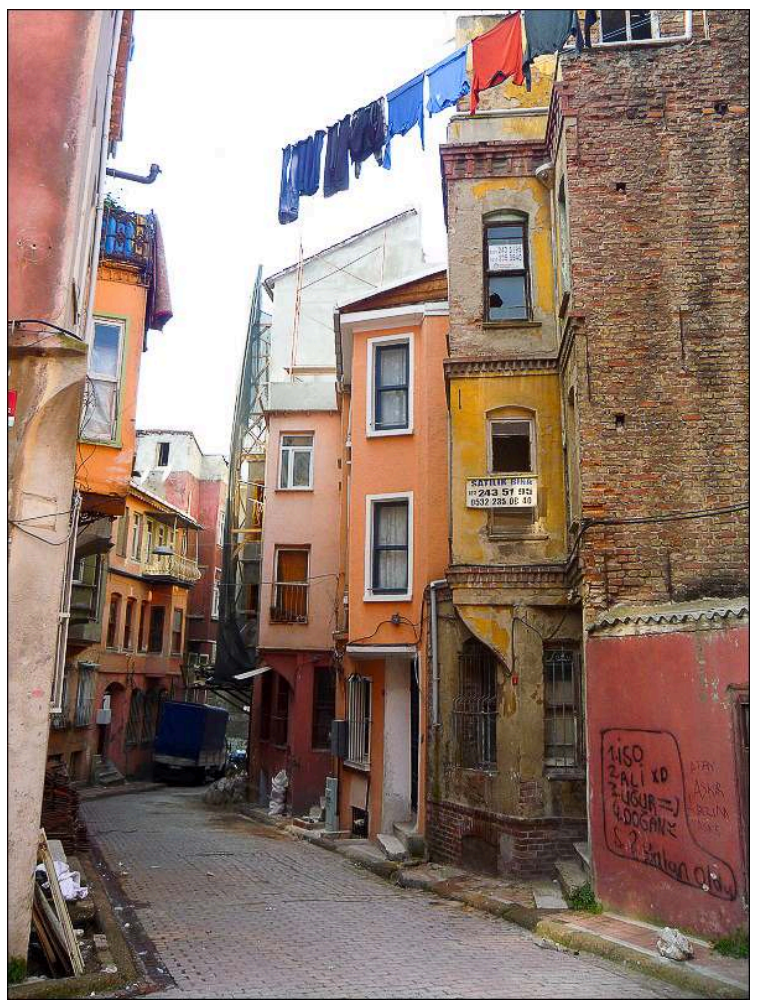

Auteur : Brian Chauvel, février 2011

10 La plupart des dernières anciennes bâtisses en bois, telles celles qui à flanc de coteaux remontaient sur Çarşamba, ont été remplacées par des constructions en béton dans les années 1970-1980. Mais elles reviennent au goût du jour. Des rénovations ou constructions neuves utilisant des bardages bois ou des imitations plaquées en polyme se multiplient en effet dès que l'on sort du périmètre classé de «Fener-Balat ", à commencer par la zone de contact avec Çarşamba. La propagation de constructions dans ce style "néo-ottoman » marque un seuil au-delà duquel on retrouve l'esthétique qui se diffuse dans les espaces rénovés de Süleymaniye, de Zeyrek et en d'autres points de la péninsule. 
Illustrations 2 et 3 - Rénovations dans le style néo-ottoman à 50 mètres du Lycée rum de Fener à proximité du centre coranique d'İsmail Ağa, Çarşamba

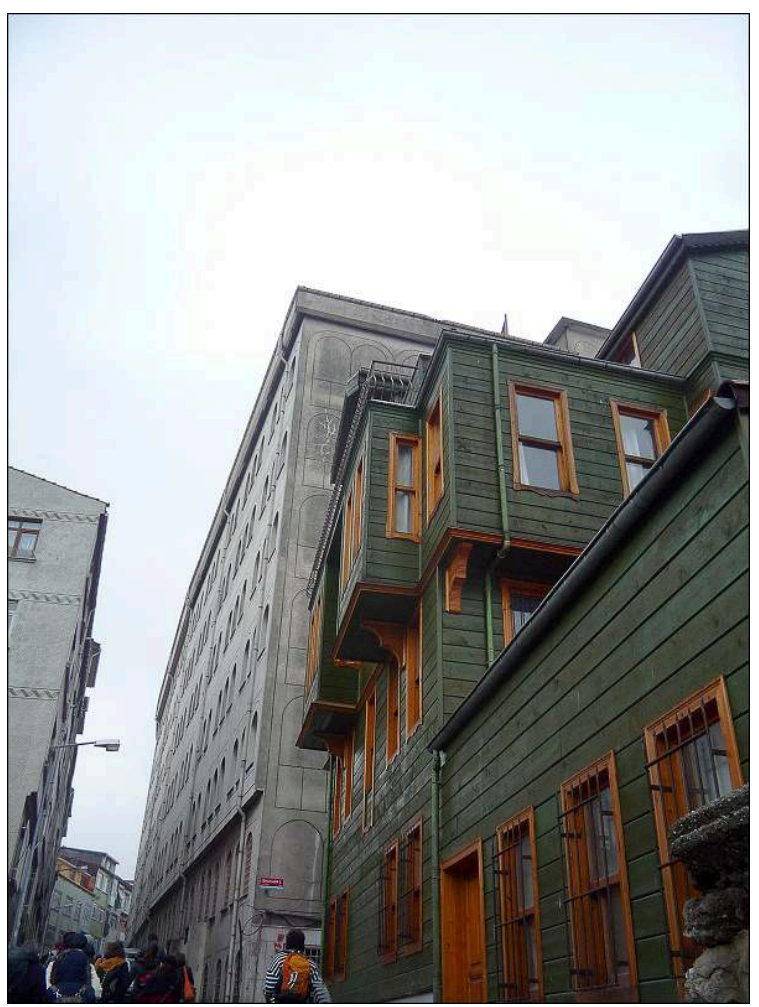

Rue İsmail Ağa, la rénovation a été menée par la « Fédération des associations donnant de la valeur à I'homme », une antenne du centre coranique qui s'investit dans des œuvres humanitaires en Guinée et au Pakistan.

Auteur : Brian Chauvel, février 2011 


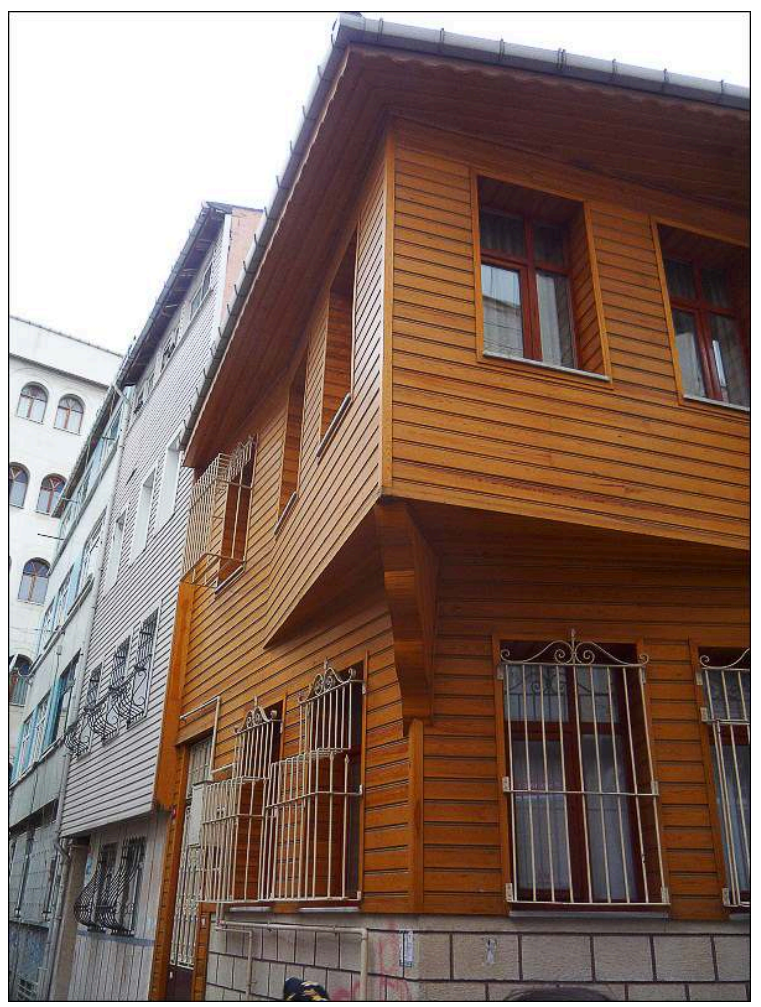

Rue Mescidi, la rénovation a été menée par un particulier Auteur : Brian Chauvel, février 2011.

La frontière formée par le dénivelé entre Fener et Çarşamba se trouve ainsi renforcée par une distinction architecturale. Or, derrière celle-ci, s'en profile une autre, confessionnelle. Tandis que Fener abrite le siège du Patriarcat œcuménique grec orthodoxe, Çarşamba est dominé par l'imposante mosquée de Sultan Selim, père de Soliman, honoré pour avoir lutté contre l'« hérésie » safavide et « imposé le califat face au Shah d'Iran $»^{5}$. Une des trois branches stambouliotes de la confrérie Nakşibendiye est implantée à proximité, dans la mosquée İsmail Ağa et possède un important centre coranique (éponyme). Depuis deux à trois ans, cette communauté a intégré une architecture d'inspiration néo-ottomane à son répertoire de signes distinctifs (cf. illustration 2). Cet usage de l'architecture civile, qui s'inspire des processus de patrimonialisation en donnant au neuf des allures d'ancien, pourrait être rapproché du principe de l'archive ${ }^{6}$ en ce qu'il aide à penser d'une part, les rapports entre le monumental et le civil et d'autre part, entre des différents types d'architectures conservées, restaurées ou rénovées. Plus directement, et c'est ce qui invite à qualifier l'articulation entre Çarşamba et Fener en termes de frontières et non de rupture, la patrimonialisation d'un type de bâti ayant des effets d'entrânement sur les autres.

\section{Fener : amalgame identitaire autour d'une réminiscence «byzantine»}

12 Le projet de réhabilitation mené en partenariat entre la Mairie de Fatih et l'Union européenne dans les "quartiers" de Fener et de Balat visait explicitement et techniquement «l'amélioration des conditions de vie des habitants » ${ }^{7}$. Mais il pouvait difficilement échapper à des interprétations qui lui attribuèrent des intentions cachées. 
Comme indiqué dans le rapport préalable, «Balat est connu comme un quartier juif dont l'origine remonterait à l'époque byzantine (...) mais c'est surtout après la conquête ottomane que le quartier se développa en raison des transferts de population opérés par le sultan Mehmet II dans le cadre de la politique de repeuplement de la capitale ». La brève présentation de l'histoire de Fener, quartier qui abritait notamment les demeures des drogmans de l'empire, évoque un héritage prestigieux qui rappelle aussi la présence d'une communauté non-musulmane: "le quartier devait son importance au fait qu'il constituait le centre du patriarcat grec et de l'église orthodoxe ». L'histoire de Fener et de Balat, quartiers réunis dans un même secteur de réhabilitation, peut alors sembler ancrée dans un même passé lié à la présence de populations désignées comme « minoritaires ».

Dans un contexte global de regain d'intérêt pour les quartiers anciens et bien que cela semble totalement hors des intentions des concepteurs du projet de réhabilitation, ce passé ne manque pas d'évoquer l'image idéalisée d'un cosmopolitisme radieux. Fener attire de nouveaux habitants, dont une proportion croissante d'étrangers fascinés par $l^{\prime}$ « âme $"^{8}$ des lieux. Toutefois, au regard des tendances qui se profilaient dès l'élection du Refah à la tête des municipalités d'Istanbul et de Fatih, la mise en valeur d'espaces dont l'histoire rappelle l'ancienneté, l'importance numérique et sociale de la présence juive et rum à Istanbul semble problématique. Au cours de polémiques portant sur les projets de transformation urbaine du secteur, un argument émergea contre le projet de réhabilitation lancé en 1997, argument qui ressurgit à l'occasion du projet de «renouvellement» annoncé en 2009 par la mairie de Fatih et confié à un holding (Çalik) lié au pouvoir AKP.

Document 1 - « Savez-vous que vos maisons vous glissent entre les doigts ? Touche pas à ma maison !...»

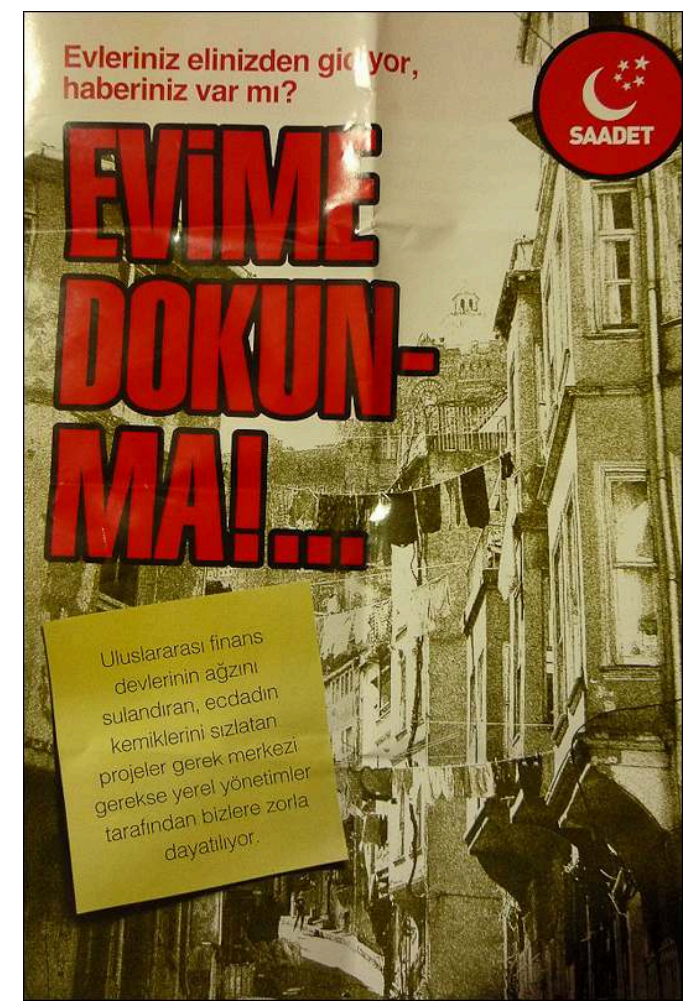

Tract du Saadet partisi, distribué dans les quartiers de Fener et de Balat en juin 2010. 
Pour expliquer les polémiques autour de ce projet, le muhtar ${ }^{9}$ supposait ainsi que, outre les intérêts des «étrangers et des riches » qui investissent dans ce secteur et ceux d'un «Etat profond» qui privilégierait un groupe dont le promoteur est lié au gendre du Premier ministre Erdoğan, ces polémiques seraient aussi nourries par une opposition entre les « islamistes d'İsmail Ağa » (à Çarşamba) et le Patriarcat de Fener. De même, un tract diffusé en juin 2010 dans les rues de Fener et de Balat par le Saadet, parti de «la félicité $»^{10}$, annonçait aux habitants du quartier que leurs maisons étaient en train de leur échapper. Selon ce tract, après avoir été cédées à des "doubles nationaux " (l'accusation semble viser des Rum ainsi soupçonnés d'intelligence avec la Grèce) lors du projet soutenu par l'Union européenne et l'UNESCO, ces propriétés seraient en effet menacées par une loi sur l'accession à la propriété des fondations religieuses. Cette loi, votée en 2008, faciliterait désormais l'emprise du Patriarcat et de "la finance internationale " sur un territoire sacré («ecdat» liant la référence religieuse à un terme ottoman).

Bien que ces théories aient un retentissement relativement faible (le muhtar ne leur accorde qu'un poids limité, et le Saadet est un parti marginal), leur émergence dans des contextes polémiques révèle le type d'enjeux qui peuvent affleurer lors d'opérations touchant le patrimoine bâti : la question du patrimoine est ici directement imbriquée dans des réseaux d'intérêts identifiés à des communautés religieuses (İsmail Ağa, rum) et lue comme répondant à des stratégies de conquête et de dépossession. Les logiques économiques, d'ouverture à "la finance internationale " (ce que le Saadet reproche à l'AKP) ou même d'échelle plus réduite peuvent ainsi être analysées comme autant de marqueurs mobilisables pour identifier et différencier des territoires subjectifs dépassant l'échelle du quartier (territoires mythiques, confessionnels et nationaux).

\section{"Byzance" et ottomanisme : traductions commerciales d'imaginaires transnationaux}

16 A Fener, la présence du Patriarcat œcuménique a une conséquence directe sur la morphologie spatiale. Situé face à l'embarcadère, mais en retrait du boulevard côtier, il draine un flux régulier de touristes qui entrent par l'ancienne porte percée dans la muraille byzantine, porte autour de laquelle s'organise un petit secteur commerçant. Le «kiosque du progrès » (Çağdaş Büfe), à l'angle du boulevard et de l'entrée de Fener, vend les mêmes cigarettes, journaux et encas que tout autre büfe. Il s'est juste attaché à proposer une spécialité détonnant avec l'offre classique de ce type d'établissement: du café frappé. La publicité faite à ce produit, en grec, cible une clientèle spécifique faisant halte au Patriarcat après avoir visité l'église des Blachernes : des groupes de touristes grecs y sont quotidiennement déchargés et récupérés par bus. Trois boutiques de souvenirs marquent aussi cet espace d'une manière similaire à celles qui s'étendent le long des rues beaucoup plus fréquentées autour du Bazar Egyptien; y sont vendus les mêmes foulards, chaussons, narguilés, amulettes contre le mauvais œil et guides multilingues. Une seule différence : ici, c'est surtout en grec qu'on attire le chaland. 
Illustration 4 - Commerce touristique à l'entrée de Fener

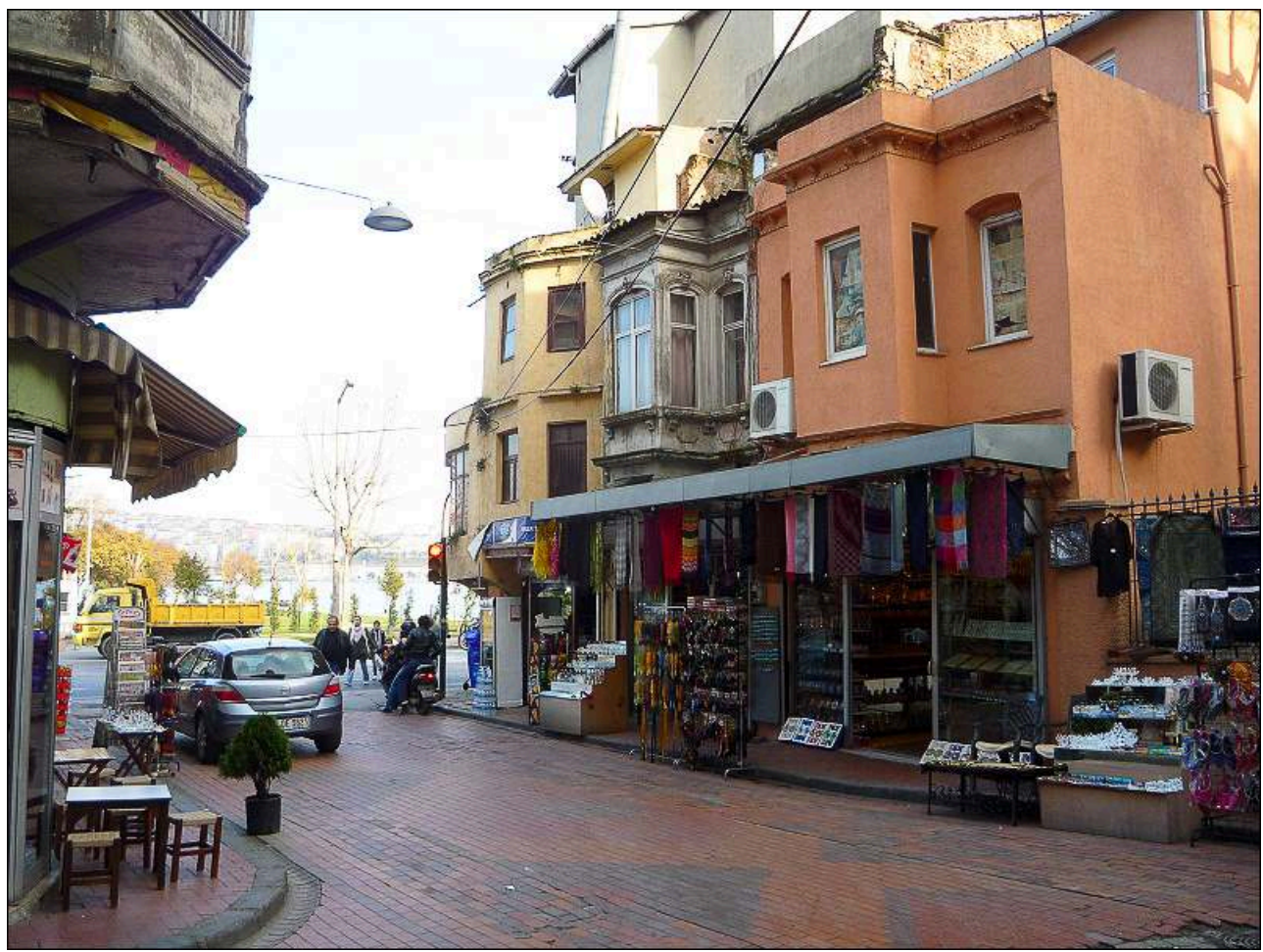

Auteur : Brian Chauvel, février 2011

Illustration 5 - Commerce touristique à l'entrée de Fener

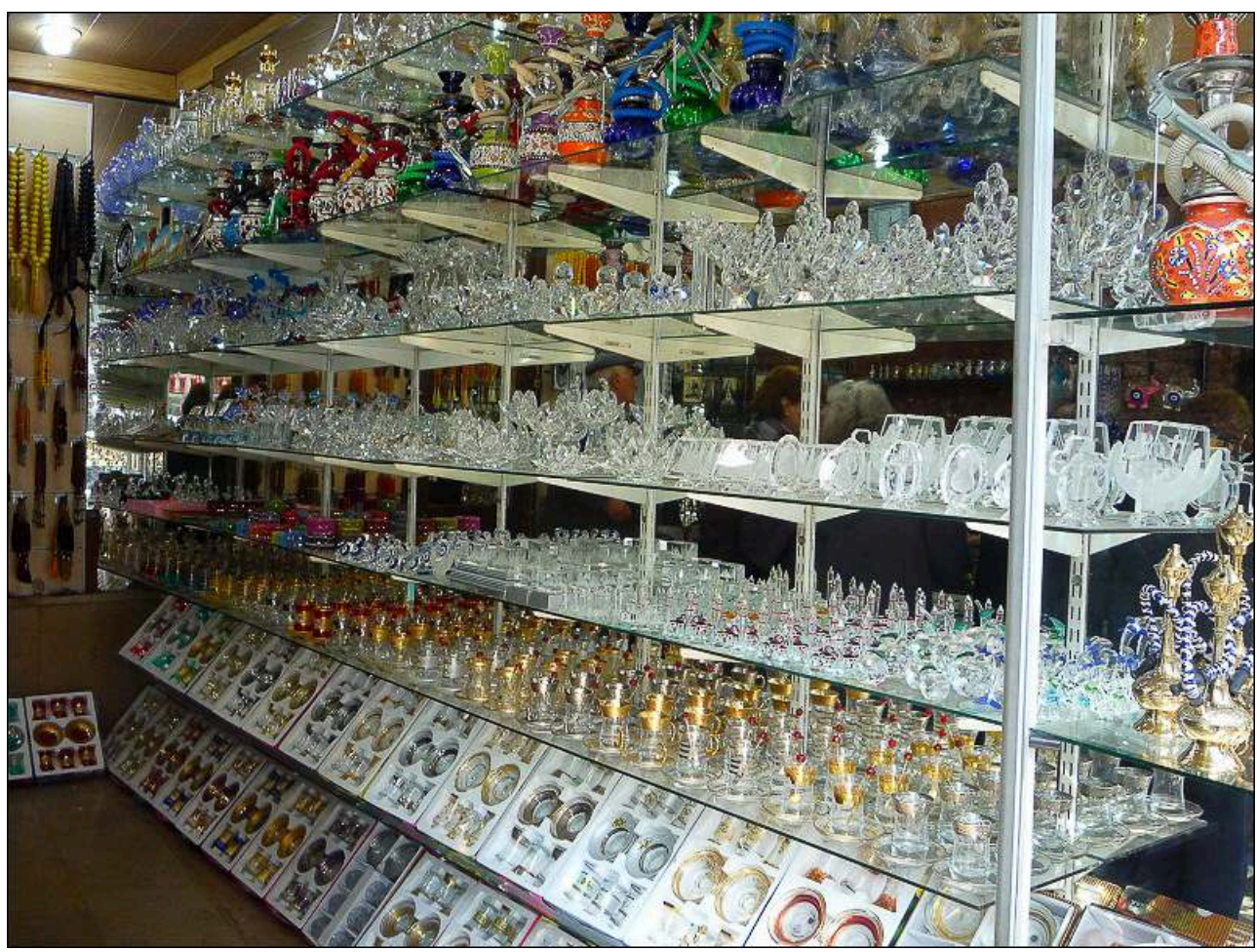

Auteur : Brian Chauvel, février 2011 
17 A quelques mètres de là, des serviettes et sorties de bains sont vendues dans l'univers chaleureux, cosy, d'une boutique nommée «BYZA $\Sigma$ ». L'offre semble alors tout autant destinée à des touristes de passage qu'à une population habitant les environs dans un intérieur confortable. La référence réapparaît trente mètres en amont de cette rue, sur la façade en mosaïque (qui rappelle l'art byzantin) d'un ancien « KAФNEION » (café) qui abrite l'association socioculturelle «Mavi Kalem», une association qui œuvre, en recourant à des volontaires internationaux, à "l'amélioration de la qualité de la vie quotidienne de beaucoup de familles du voisinage $»^{11}$. A proximité du Patriarcat, l'affichage de ces références révèle et entretient un amalgame entre des dimensions politiques, juridiques et identitaires qui s'entremêlent et s'interpénètrent ${ }^{12}$. Elles produisent un malentendu « productif ».

18 Un ancien antiquaire s'était installé à proximité, au rez-de-chaussée de la première bâtisse réhabilitée par le projet mené par l'Union européenne et la mairie de Fatih (dont les travaux débutent en 2003). Placé à l'intersection de la rue Vodina (qui court jusque Balat) et de l'axe remontant à Çarşamba, ce commerçant collectait toutes sortes d'éléments de décoration intérieure et d'ouvrages. Des lots de recueils de prières en grec et en arménien, aux reproductions d'icônes, en passant par les amulettes protectrices contre le mauvais œil, sa collection hétéroclite était à même d'apporter la touche "mystique " aux intérieurs d'un ancien semt redécouvert par de nouvelles populations. En 2010, après quelques années d'activité, cet antiquaire-accessoiriste «Kemha accessories » est devenu agent immobilier-décorateur. Après avoir constitué un carnet d'adresses conséquent auprès des étrangers rencontrés en ce point de passage, «Kemha emlak dekorasyon" s'est ainsi lancé dans cette "la guerre de l'immobilier $»^{13}$ qui oppose un chapelet d'agences distribuées le long de la rue reliant ce carrefour à l'entrée touristique de Fener. Au vu des prix affichés dans ces agences immobilières (loyers de $400 €$ les $35 \mathrm{~m}^{2}$ ) ${ }^{14}$, l'espoir de réaliser des plus-values de l'ordre de celles opérées dans les quartiers gentrifiés de Beyoğlu est effectivement explicite. 
Illustrations 6 et 7 - Ancien antiquaire devenu agent immobilier, Fener

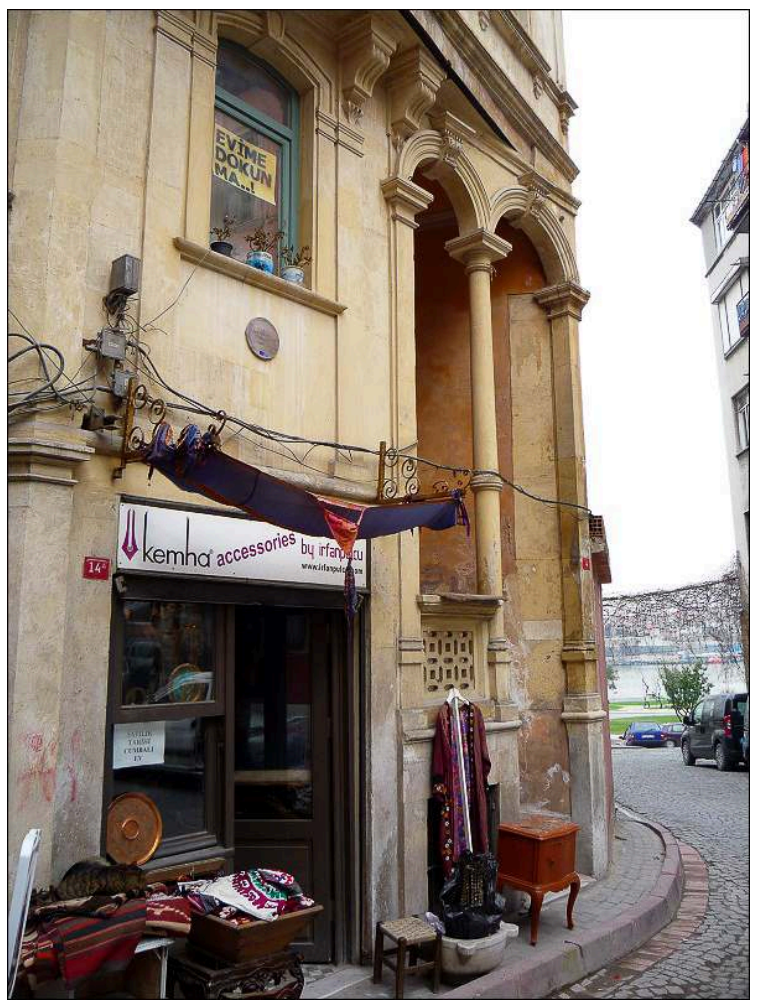

Auteur : Brian Chauvel, avril 2010

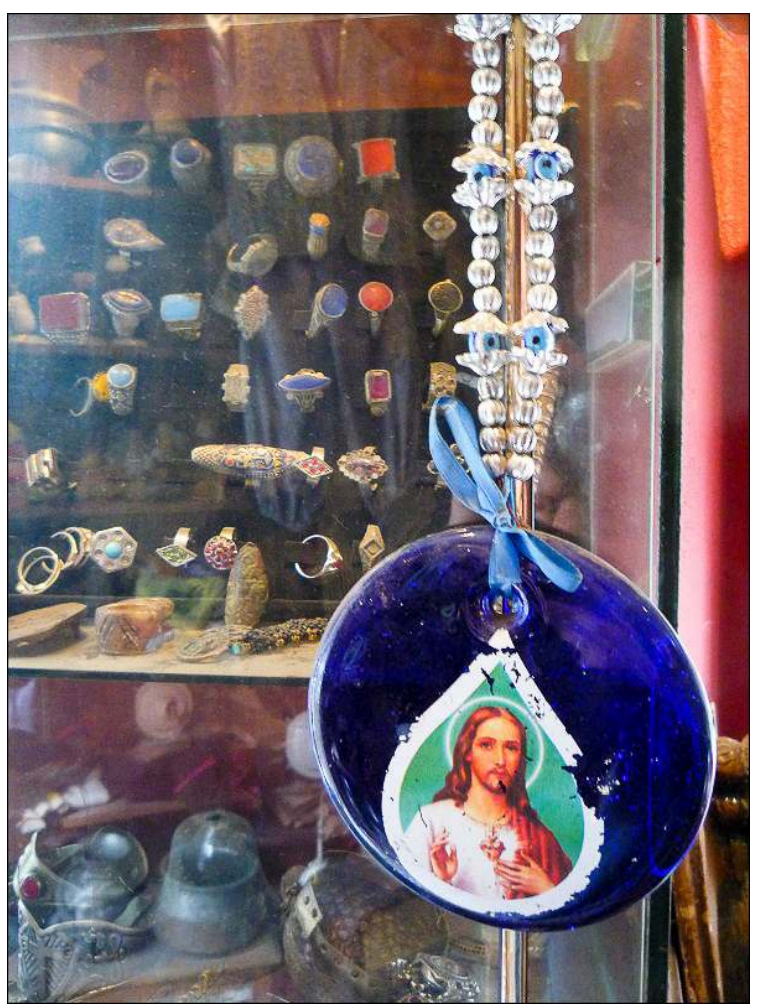

Auteur : Brian Chauvel, avril 2010

19 Cette double implantation commerciale, étalée sur deux carrefours situés à 50 mètres de distance, connecte Fener à deux types d'espaces. L'un, dans lequel évoluent les 
touristes arrivant par bus depuis l'église des Blachernes (située 500 mètres en amont de la Corne d'Or, à Ayvansaray) est ancré dans une mémoire religieuse et nationale d'une population rum devenue minoritaire après les années 1950 (au fil des « événements » du 5-6 septembre 1955, de 1964 et 1974). L'autre, identifiable à l'implantation d'étrangers (et, pourrait-on rajouter, «de riches») sensibles à «l'âme» des lieux, participe d'un imaginaire global inspiré par le mythe du village urbain peuplé de voisins vivant dans une atmosphère conviviale, mythe qui ne s'éloigne pas beaucoup de l'image diffusée sur les ondes nationales (et internationales) par des séries télévisées, telle "Yeditepe Istanbul » (2001-2003) où est mise en scène l'arrivée d'une bourgeoise déchue, prise en charge par un voisinage populaire. Le tout, bien que connecté à Balat par la rue Vodina semble s'en détacher; l'implantation commerciale sur cet axe, limitée à quelques épiceries et ateliers, est plus clairsemée durant une centaine de mètres. Mais la distance n'est pas ici aussi prononcée qu'avec Çarşamba, frontière que les visites guidées de Fener ne franchissent jamais.

\section{Çarşamba et la commercialisation de la piété}

Çarşamba résonne, dans l'imaginaire collectif de certaines classes sociales (particulièrement celles à qui s'adressait Oktan Ekinci), comme le lieu par excellence du rigorisme religieux. Les barbes y sont longues, la tenue vestimentaire codifiée; la plupart des femmes entièrement de noir vêtues, les hommes à l'image ${ }^{15}$ du Cüppeli Ahmet Hoca - à la tête de la confrérie İsmail Ağa -, en longue tunique coiffés d'un turban. Ce lieu commun se projette dans un espace marqué par les mosquées İsmail Ağa et Sultan Selim et un imposant centre coranique, qui dominent la Corne d'Or et se dressent au dessus de Fener. Cet espace est traversé par l'avenue commerçante Manyasizade caddesi qui parcourt une ligne de crête se prolongeant par l'avenue Darüşşafaka caddesi jusqu'au complexe de Fatih, symbole éponyme du conquérant. Une forte concentration de boutiques d'habillement et d'ateliers de confections spécialisés dans le vêtement pieux (dua giyimi) ainsi que de librairies et maisons d'éditions spécialisées dans les publications islamiques (livres religieux, méthodes d'apprentissage de l'arabe, conseils matrimoniaux,...) marque la spécificité commerciale de ce secteur. L'influence de la mosquée İsmail Ağa, dont les fidèles ont des critères bien définis en termes vestimentaires et religieux, est certaine. Ces lieux n'en sont pas moins ouverts, étendus à un espace mythique et contemporain, marqué par le Livre, scellé dans des mausolées de personnages dont la piété inspire le respect aux pèlerins qui se recueillent quotidiennement sur leur tombe (tel le türbe de Mustafa Ismet Efendi, rouvert en 1960 puis restauré par la Nakşibendiye). 
Illustration 8 - Commerce spécialisé à Çarşamba, rue Manyasizade

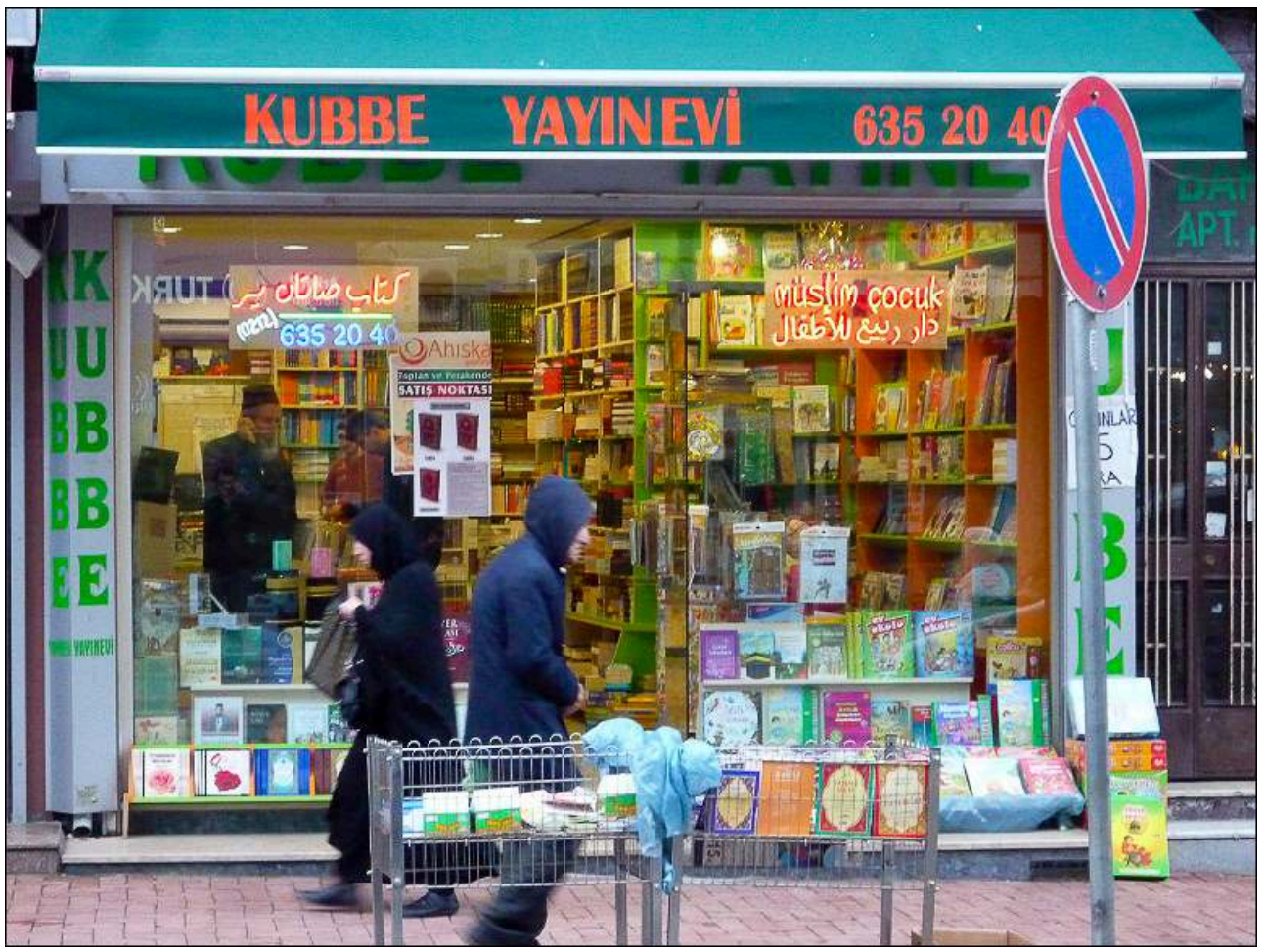

Auteur : Brian Chauvel, février 2011

Illustration 9 - Commerce spécialisé à Çarşamba, rue Manyasizade

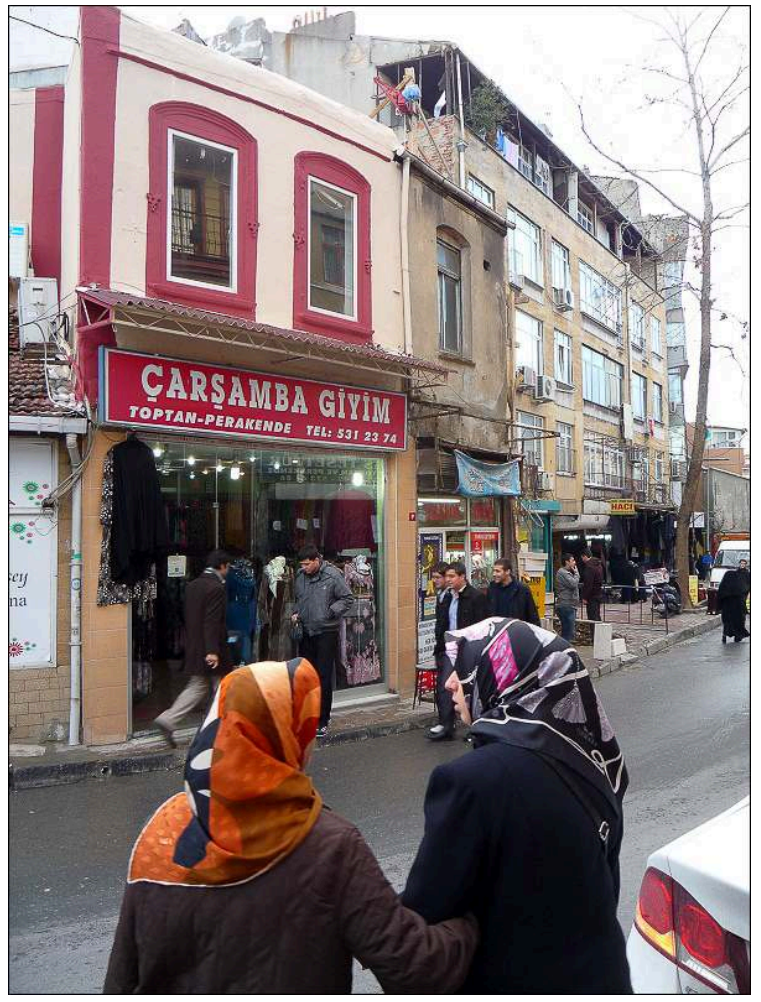

Auteur : Brian Chauvel, février 2011 
Etant dans le prolongement d'un axe qui abrite un très important marché chaque mercredi (çarşamba en turc) et le relie à la mosquée Fatih, l'espace commercial de ce quartier s'inscrit dans une continuité relative avec un espace sacralisé. Les récents centres commerciaux de l'avenue Darüşşafaka caddesi (qui débute au pied de la mosquée de Fatih) se distinguent cependant des petites boutiques de Çarşamba. Après BíM (enseigne liée au maire AKP d'Istanbul), l'installation de «Selam Hipermarket » et l'ouverture récente (le 25 février 2011) de "Berat Lüks Hipermarket " relèvent de projets nécessitant d'importants investissements financiers qui ciblent une classe moyenne consommatrice de produits hallâl. L'esthétique affichée sur ces immeubles de commerce en rez-de-chaussée et d'appartements sur les trois ou quatre étages supérieurs, enchevêtrant ces références islamiques à des références anatoliennes, reflète une évolution du récit et de l'identité nationale donnant plus de poids aux tenants de la «synthèse turco-islamique » durant les années 1990 (Copeau, 1998). Elle peut aussi donner l'image des trajectoires sociales et résidentielle d'habitants particulièrement réceptifs à ces thèses.

Illustration 10 - Développement d'une grandes surface Berat entre Çarşamba et la mosquée de Fatih, rue Darüşşafaka

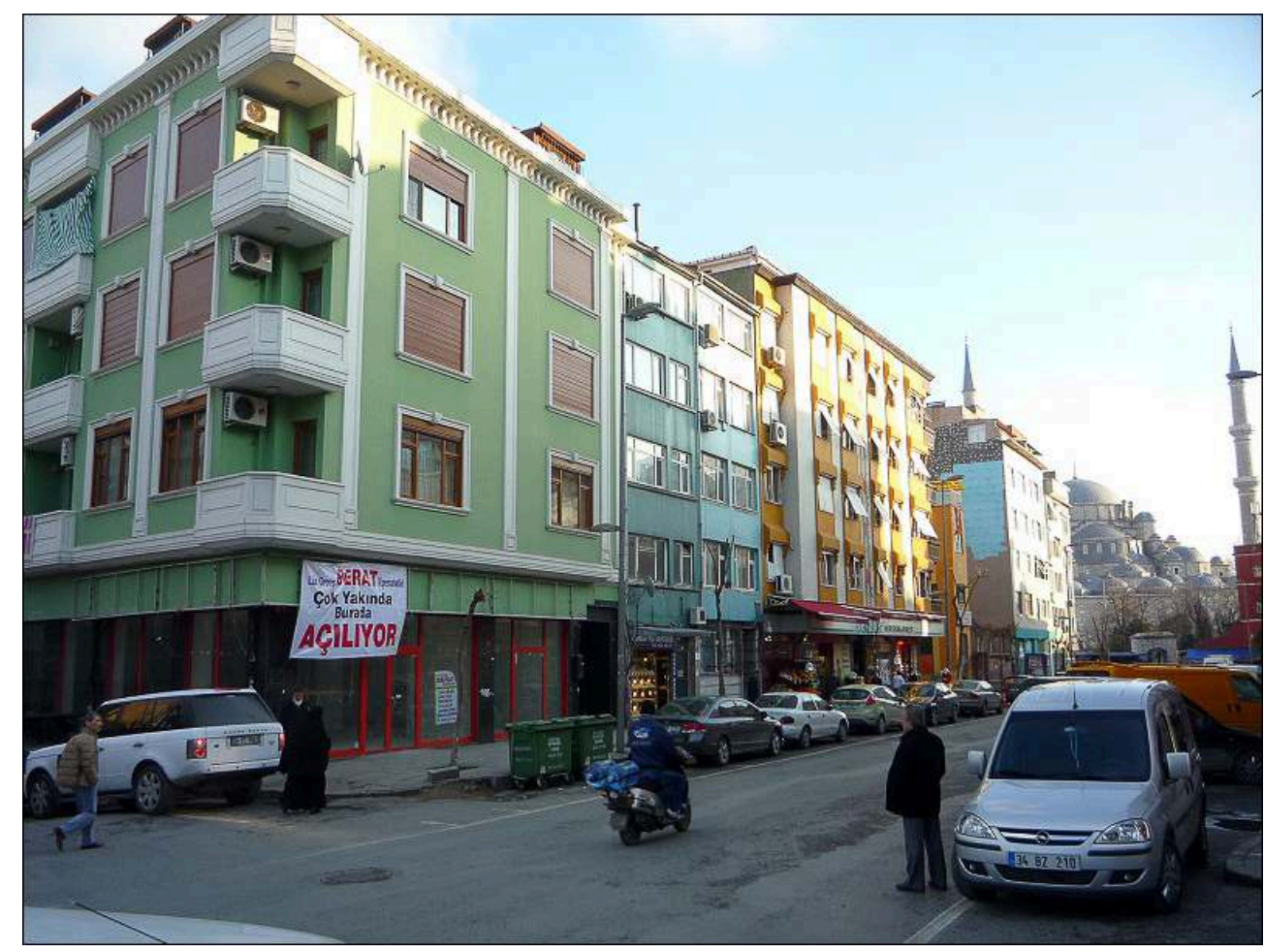

Auteur : Brian Chauvel, février 2011 
Illustration 11 - Développement d'une grandes surface Selam entre Çarşamba et la mosquée de Fatih, rue Darüşşafaka

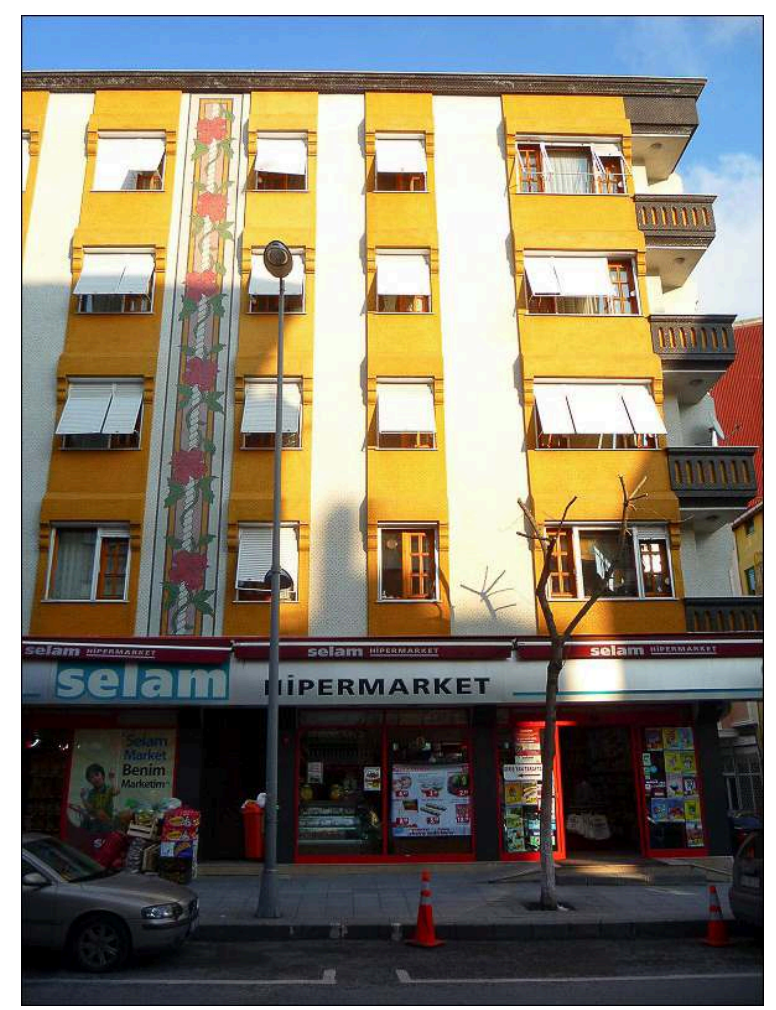

Auteur : Brian Chauvel, février 2011.

Mais cette esthétique varie : entre cet axe et celui de l'avenue Fatih caddesi, dans la ruelle Başhoca, où se trouve l'ancien bâtiment d'une fondation pieuse « au service de la connaissance et la vertu » (fatih ilim ve fazilete hizmet vakfi) et le bureau de la section d'arrondissement du saadet partisi, se sont élevées des résidences de «lüks» aux couleurs quelque peu "tape à l'œil», dont les noms à résonance religieuse (telle la résidence derviş), s'appliquent sur des façades épurées des motifs anatoliens. Ceux-ci sont remplacés par des moulures en plâtre évoquant un bardage de type «ottoman ». L'ottomanisme appuie ici une distinction sociale (ou du moins une aspiration) qui aménage une distance envers des références plus "traditionnelles» (symbolisées par les motifs du kilim). Passant de la mosquée de Fatih à Çarşamba, on peut donc observer non seulement comment un espace marqué par des références islamiques articule un idéal de consommation à un idéal de piété, mais aussi que la convocation d'un idéaltype (néo)ottoman est porteur d'une distinction mettant en avant une identité citadine (le lüks s'opposant à la référence anatolienne). 
Illustration 12 - Centre coranique (en travaux) de la « Fatih ilim ve fazilete hizmet vakfı »

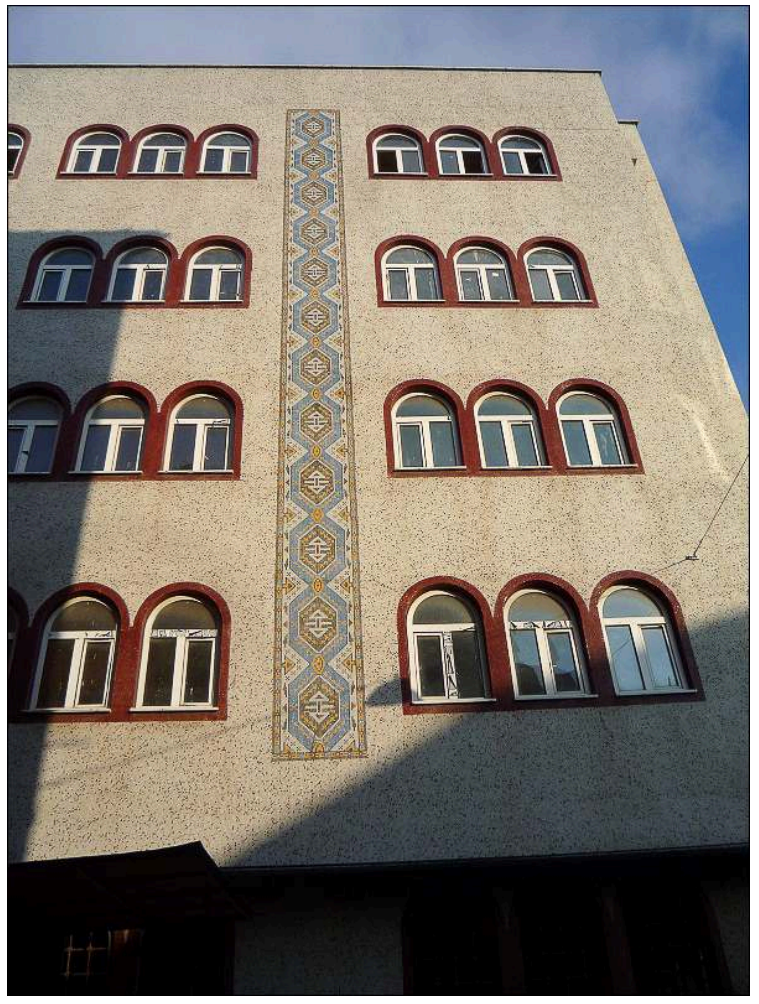

Auteur : Brian Chauvel, février 2011

Illustration 13 - Résidence de « lüks » entre le siège du Saadet et le centre coranique, rue Başhoca

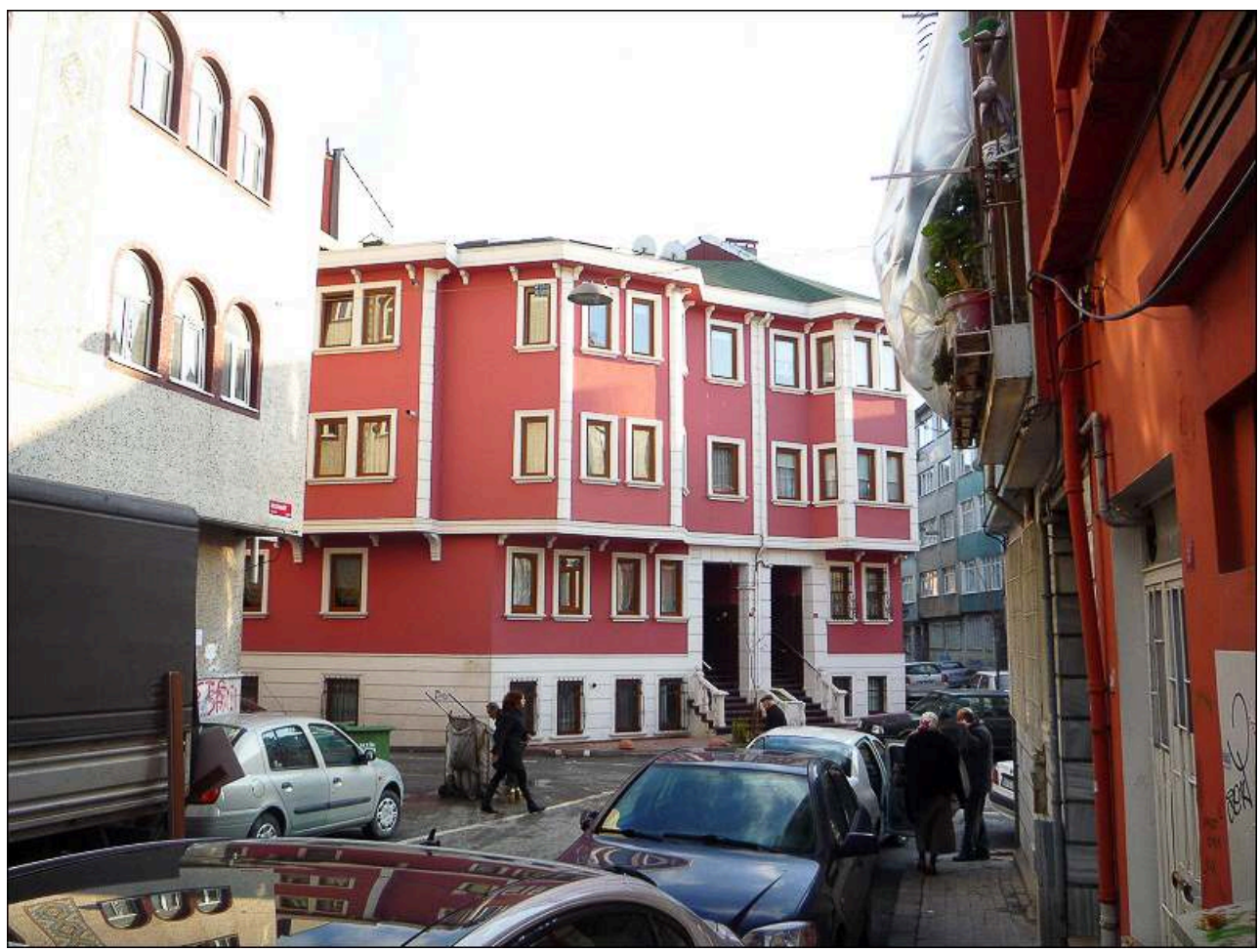

Auteur : Brian Chauvel, février 2011. 


\section{Sultan Selim et la moralisation de l'espace public}

La mosquée de Sultan Selim surplombe le Patriarcat Rum depuis un belvédère qui, offrant une magnifique vue sur la Corne d'Or, est aussi fréquenté pour son panorama. Espace de coexistence de ces deux logiques de fréquentation, l'une attachée au culte, l'autre au paysage, le parc entourant cette mosquée est l'objet d'une vigilance toute particulière: chaque lampadaire est porteur d'un message rappelant au visiteur les règles qui se doivent d'être observées. Cette fièvre signalétique, s'attaquant aux piqueniques, jeux de balles et vélos, proscrivant l'abandon d'animaux ou même le simple fait de les nourrir et interdisant de marcher sur les pelouses, imprime ainsi aux usagers une discipline qui s'applique aussi dans le domaine du culte. Le mausolée de Sultan Selim adjacent à ce parc est en effet pris dans un dispositif tout aussi didactique, qui encadre strictement les pratiques de dévotion. Protégé par vidéosurveillance en intérieur et extérieur, gardé par un agent de sécurité qui y lit ostensiblement le Coran, le « neuvième sultan ottoman et le soixante-quatorzième calife des musulmans ${ }^{16} \mathrm{y}$ est honoré pour avoir défait le Shah d'Iran à Çaldiran et rendu « un grand service à l'Islam » en prévenant « la diffusion de fausses croyances». Projeté et retraduit dans l'espace contemporain le principe d'orthodoxie appelle, en retour, à la civilité. Sur le promontoire de la mosquée, deux pancartes s'adressent aux amoureux des bancs publics : «Comportons-nous moralement » (Ahlaka uygun davranalım)!

Illustrations 14 et 15 - Affichage public sur le promontoire de la mosquée Sultan Selim

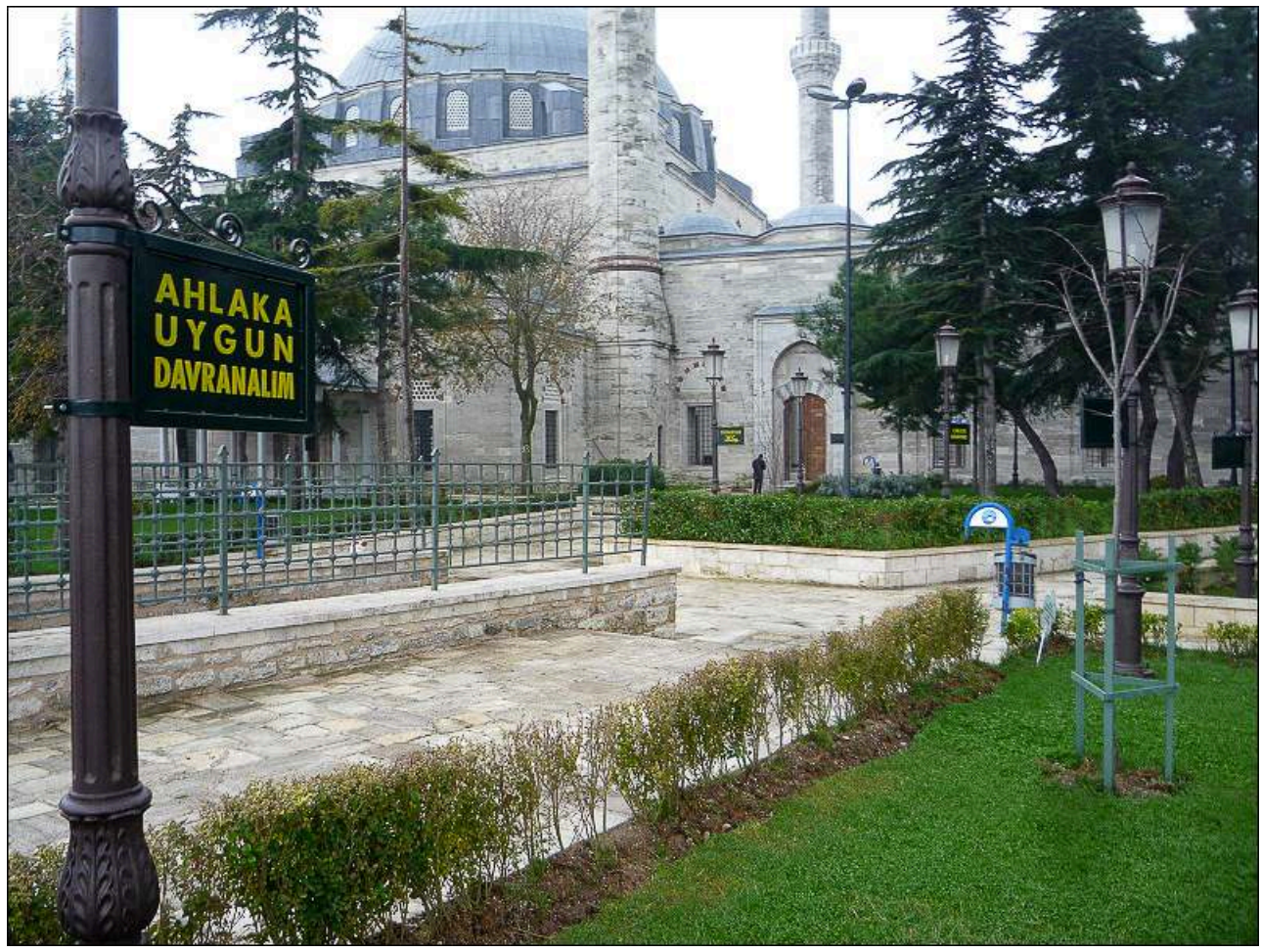

Auteur : Brian Chauvel, février 2011 


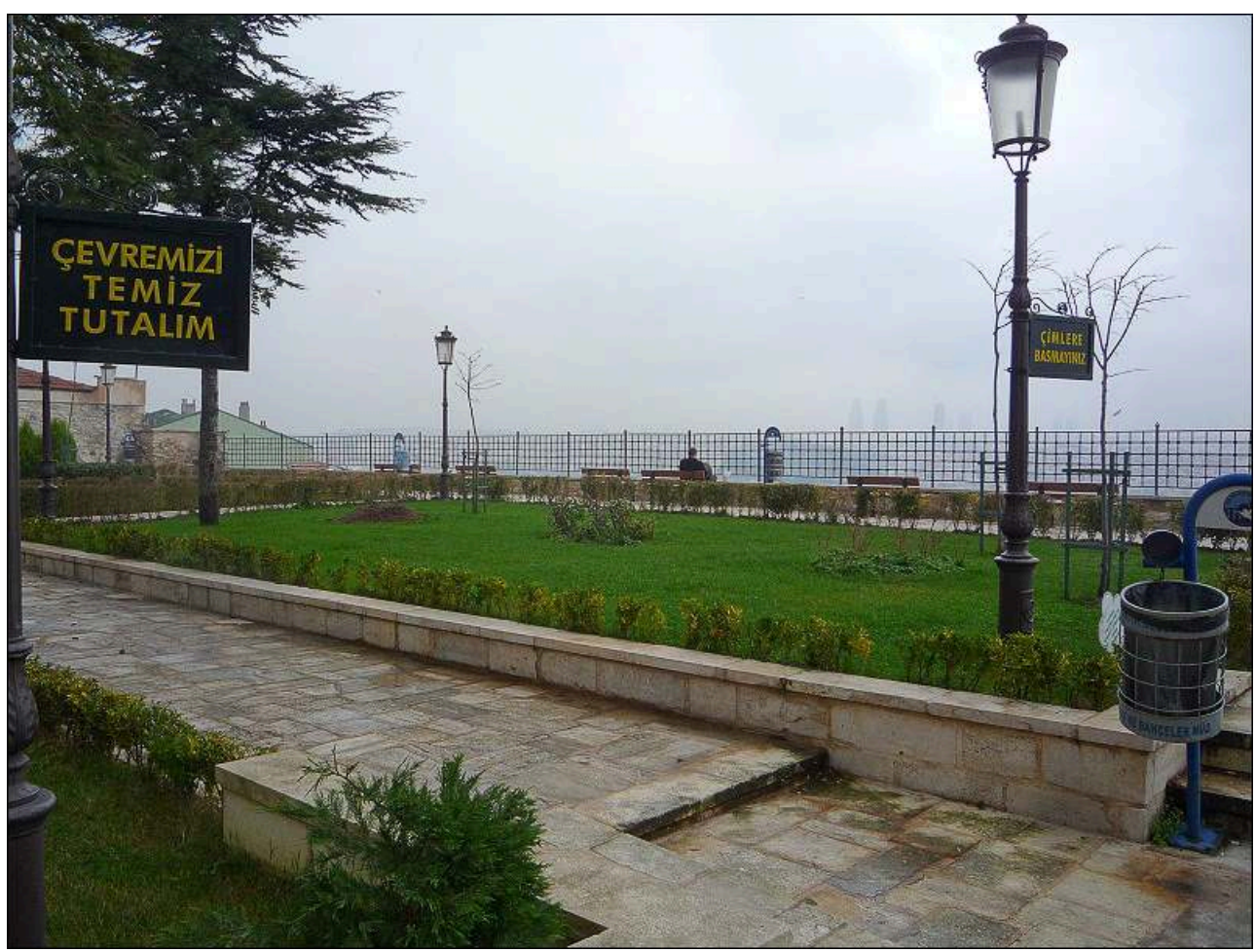

Auteur : Brian Chauvel, février 2011

\section{Entre Fener et Çarşamba, un lien touristique?} passages furtifs par la rue Manyasizade, au cours d'un trajet qui peut mener les visiteurs plus aventureux de la mosquée de Fatih, voire celle de Sultan Selim, vers l'ancienne église byzantine Saint Sauveur in Chôra (Kariye Cami) - un exemple précurseur d'aménagement urbain autour d'un secteur patrimonialisé sur une initiative du Touring et Automobile Club de Turquie dès 1985-1986. L'église Saint Sauveur étant située hors de Çarşamba, elle n'y génère qu'un faible flux de badauds qui ne s'attardent pas dans le quartier, ceux-ci se contentant souvent de descendre le long de la muraille terrestre, pour éventuellement rejoindre l'église des Blachernes ou Balat. Les restaurations de l'ancienne église Pammakaristos (Fethiye Cami), récemment ouverte au public sous forme de musée, et de la bibliothèque du Molla Murat entreprise par la mairie de Fatih et «Istanbul 2010 », à laquelle il faut ajouter celle du complexe attaché à la mosquée Sultan Selim, dont la mairie finance une partie des travaux, sont les seules initiatives pouvant $a$ priori attirer ce genre de public. Mais, à ce jour, elles n'ont pas entraîné de mutations commerciales significatives dans leurs alentours. Les prix de l'immobilier n'ont pas, non plus, suivi l'évolution enregistrée à Fener; les agences de Çarşamba affichent des loyers deux à trois fois inférieurs.

Cependant, la réunion, en 2009, de ces deux quartiers dans une même subdivision administrative incite à la comparaison et laisse penser qu'il y aurait une volonté de mieux les lier. Le muhtar porte ainsi un œil attentif sur Fener. S'il pense que les visiteurs sont principalement intéressés par le patriarcat, il met en avant l'image d'un cosmopolitisme symbolisé par la coprésence de minarets et d'institutions rum, l'imposant «Rum lisesi » ayant tendance à être pris pour le Patriarcat (cf. document 2).

EchoGéo, 16 | 2011 
La frontière où les visites guidées de Fener marquent le pas peut ainsi être pensée en termes d'articulation entre deux espaces auparavant perçus dans leur confrontation. La sécurité du quartier est un argument clé appuyant la thèse d'une entente cordiale ; l'intérêt qu'on lui a porté lui a ainsi paru encouragé par la publication dans le bulletin municipal d'un article intitulé «Balat, le quartier le plus sûr et paisible d'Istanbul » ${ }^{17}$. Les travaux de voirie menés en mai 2011 dans la rue qui descend depuis la mosquée de Sultan Selim jusqu'au Patriarcat pourraient encourager cette interprétation; si la première rue repavée dessert uniquement les résidences en contrebas, les raccroche à Carşamba et renforce la frontière physique qui les sépare de Fener (illustration 14), le repavage du fort dénivelé menant à la Corne d'Or favorise l'ouverture de Çarşamba sur cet axe.

Document 2 - Extrait de l'article « Balat, le quartier le plus sur et paisible d'Istanbul »

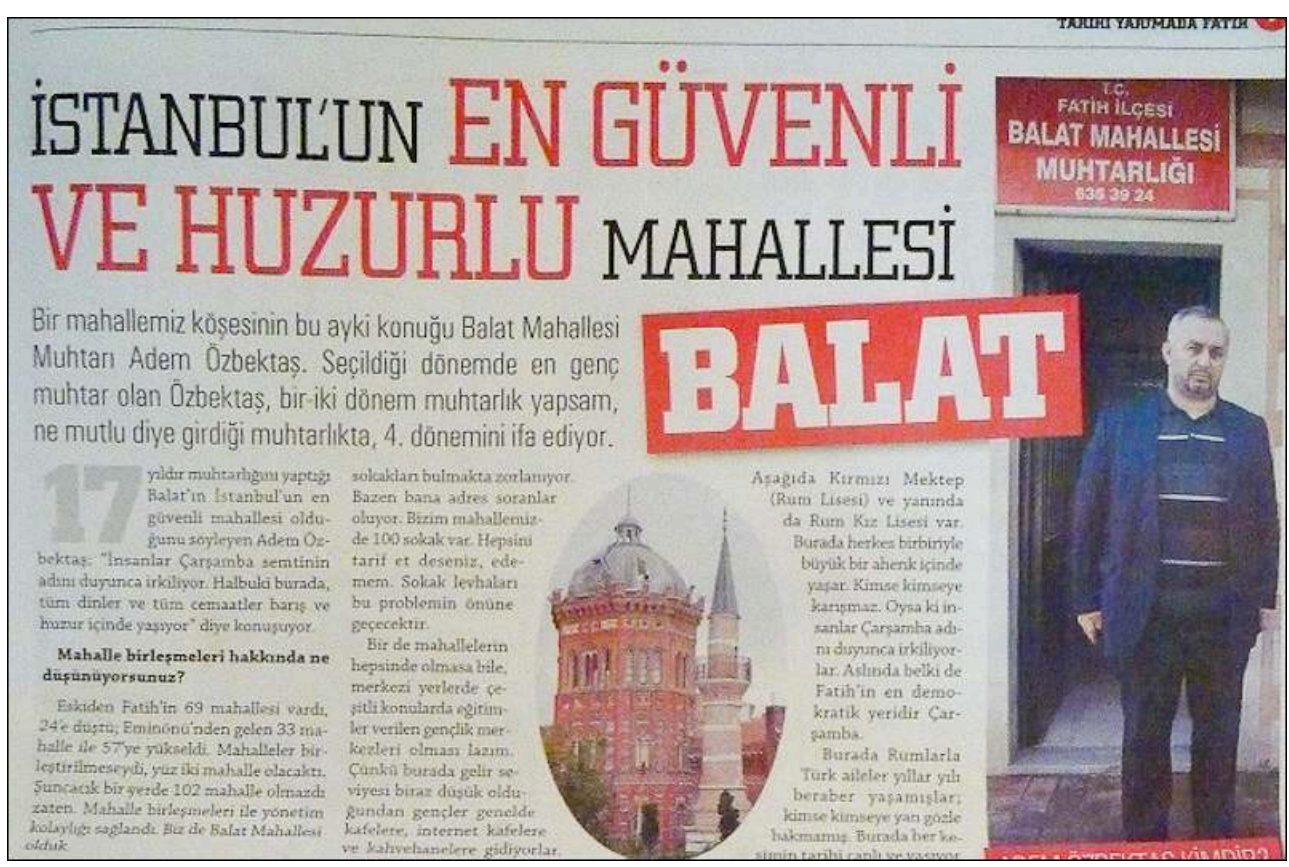


Illustration 16 - Le minaret de la mosquée Abdi Subaşı se dressant au dessus de l'enceinte gardée du Patriarcat

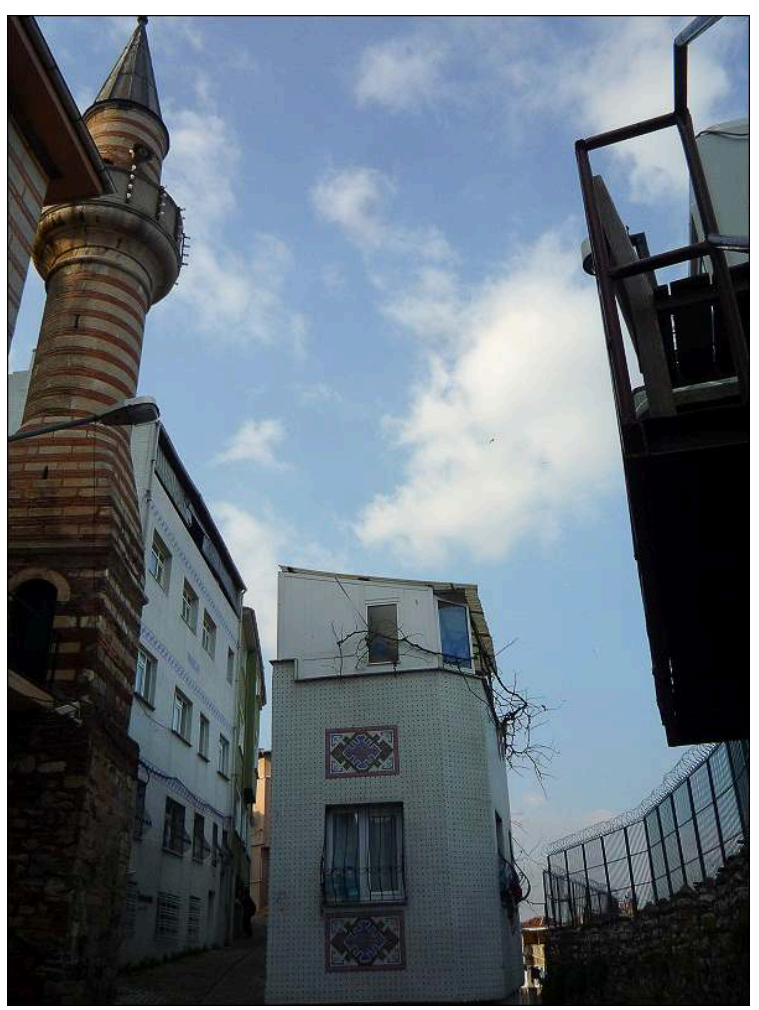

Auteur : Brian Chauvel

\section{Conclusion}

Le processus d'internationalisation d'Istanbul, tout en forgeant l'image d'une ville globale, accompagne une recomposition de l'imaginaire national. Le paradigme de la Conquête, repris par une idéologie mariant libéralisme économique et conservatisme politique, apparaît alors comme un pilier autour duquel se réorganise, particulièrement dans la péninsule historique, l'espace symbolique et social de classes moyennes s'identifiant à un idéal islamique et national. Fatih, incarnant la réalisation d'une prophétie remémorée lors des cérémonies du 29 mai, est honoré comme l'ancêtre éponyme de ce cadre socio-spatial. Ancêtre qui, non seulement rappelle l'efficacité et les fondements théologiques de l'alliance entre l'Islam et la nation turque, mais apparaît aussi désormais comme la figure tutélaire d'une civilité islamique et turque érigée en modèle d'intégration à la vie urbaine. En ce sens, la mise en valeur d'un patrimoine «ottoman» et l'inflation de constructions dans un style assimilé (« néoottoman ») ne témoignent peut-être pas d'un changement radical de paradigme, mais tout au moins d'une plus grande distance envers un modèle «occidental» et les élites qui l'incarneraient. Or cette distance s'affirme précisément par une prétention à transcender les clivages existants, à les reformuler.

Les cas de Fener et de Çarşamba sont des exemples permettant de se faire une idée circonscrite de la façon dont ce discours peut être ajusté à l'histoire nationale, aux pratiques religieuses et à des mutations économiques. Fener, en tant qu'ancien quartier des élites rum de l'Empire, non seulement conteste la thèse de la turcité d'un empire 
musulman, mais rappelle aussi le contexte dans lequel la péninsule historique s'est repeuplée depuis les années 1950 conjointement aux départs de populations "minoritaires", vers d'autres quartiers ou d'autres pays. La mise en valeur du patrimoine civil peut donc d'autant plus paraitre suspicieuse qu'elle attire de nouveaux habitants et a des effets compromettant l'installation des résidents actuels. La théorie d'un complot fomenté par des agents doubles et la finance internationale illustre comment ces questions identitaires (confessionnelles et nationales) sont renforcées par des enjeux fonciers. Dans cette perspective, le développement d'un style "néoottoman" à Çarşamba pourrait être pensé sur le mode d'une opposition avec un patrimoine qui serait «minoritaire » à Fener, ce qui appuierait la thèse d'un clivage ou d'une concurrence entre ces deux quartiers, deux conceptions du patrimoine et deux types d'usagers. Pourtant, et ce d'autant plus facilement qu'ils font désormais partie d'une même unité administrative, il semble qu'il faille plutôt s'attendre au développement d'une articulation entre l'un et l'autre. Observer l'amélioration des chaussées, le développement de commerces spécifiques autour du patrimoine monumental et religieux incite à comparer les boutiques de Çarşamba, qui proposent des produits et services pour le pèlerinage à la Mecque, à celles de Fener. Aussi, l'image de rigorisme qui est attachée à Çarşamba, notamment en raison des pratiques distinctives du groupe d'ìsmail Ağa, mérite d'être observée dans la continuité avec les implantations commerciales et résidentielles qui relient ce quartier à la mosquée de Fatih; le commerce nourrit et se nourrit des pratiques religieuses. Il alimente cependant d'autres types de distinctions, sociales, telles que celles qui s'observent à travers l'affranchissement des références "traditionnelles" anatoliennes et le développement d'un style architectural épuré, signe de «lüks». Le style qualifié de « néo-ottoman » semble alors lié à une conception du prestige et d'une civilité qui, se diffusant dans l'espace public, se pose en garant de bonnes mœurs et de sécurité. Or, si elle peut séduire les touristes et les aspirants à cette forme de prestige, la propagation de cette forme architecturale éloigne à coup sûr les plus "sales $»^{18}$, la destruction de leur habitat aidant. Le discours sur l'origine reflété par les références ottomanes trouverait ainsi sa vérification non en amont, mais dans les conséquences qu'il cautionne.

\section{BIBLIOGRAPHIE}

Cankat A., 2011. Istanbul, ville multiple; empruntes architecturales et urbaines des communautés, du XIXe au milieu du XXe siècle. Paris I, Thèse de doctorat en histoire de l'art, Poulot D (dir.), 1087 p. Copeau E., 1998. Espaces et temps de la nation turque ; Analyse d'une historiographie nationalise (1931 1993). Paris, CNRS, 369 p.

Çinar A., 2001. National History as a Contested Site: The Conquest of Istanbul and Islamist Negotiations of the Nation. Comparative Studies in Society and History, vol. 43, No. 2, pp. 364-39.

Göle N., 2003. Musulmanes et Modernes. Voile et Civilisation en Turquie. Paris, La Découverte, 190 p. 
Göle N., 2000. Snapshots of islamic modernities. Daedalus, « Multiple Modernities”, vol. 129, n 1, pp. 91-117

Mantran R., 1989. Histoire de l'Empire ottoman, Paris, Fayard, 810 p.

Pérouse J.-F., 2003. La muraille terrestre d'Istanbul ou l'impossible mémoire urbaine. Rives méditerranéennes, $\mathrm{n}^{\circ}$ 16, pp. 27-44.

Pérouse J.-F., 2010. Istanbul depuis 1923 : la difficile entrée dans le XXe siècle ? In Monceau N (dir.), Istanbul ; Histoire, promenades, anthologie, dictionnaire, Paris, Laffont, pp. 231-291.

Gürsoy D. et Hüküm U., 2006. Istanbul, émergence d'une société civile. Paris, Autrement, 248 p.

Renou L., 2010. Le projet de rénovation de Süleymaniye entre intervention urbaine et politique : la mise aux normes d'un quartier au service d'enjeux identitaires et internationaux. Mémoire de Master, Université Paris-Est Marne-la-Vallée, Institut Français d'Urbanisme - OUI, 135 p.

Şeni N., 2010. La tentative néo-ottomane et la rénovation du quartier de la Süleymaniye. Urbanisme, « Istanbul », $\mathrm{n}^{\circ} 374, \mathrm{pp} .41-42$.

Sourdel D., 1978. Article « Khalifa ». In Encyclopédie de l'Islam, Paris, Maisonneuve et Larose, pp. 975-981.

Stoquart R., 1997. Projet de réhabilitation des quartiers de Balat et de Fener ; diagnostic et premières orientations opérationnelles. Rapport intermédiaire. Mairie de Fatih, Union Européenne, centre du patrimoine mondial de l'UNESCO, $61 \mathrm{p}$.

Teboul N., 2002, Patrimonialisation et projet urbain en centre-ancien. Étude des quartiers de Balat et Fener à Istanbul. Institut d'Urbanisme de Paris - OUI.

Veinstein G., 2002. Pour une généalogie du califat ottoman. Cours au Collège de France,

Yerasimos S., 2000. Istanbul, la naissance de la ville ottomane. In Nicolet C., Ilbert R. et Depaule J.C. (dir.), Mégapoles méditerranéennes, Paris-Rome, Maisonneuve et Larose pp. 398-417.

\section{NOTES}

1. Oktay Ekinci, 1998. The complexes that threaten our health. Dailynews-Hurriyet, édition du 8 mars 1998.

2. Les commémorations de la Conquête (cf. supra) actualisent annuellement cet héritage en privilégiant un ancrage dans une histoire ottomane perçue comme islamique. La prière qui les a inaugurées le matin du 29 mai 2011 devant le tombeau du Sultan Mehmet II contribue par exemple à renforcer cet ancrage aux dépends des strates historiques co-existantes : la mosquée de Fatih, puis le tombeau du conquérant, bâtis à l'emplacement de l'ancienne église des Saints Apôtres et du tombeau de Constantin marquent pourtant, ne serait-ce que symboliquement, une continuité avec l'Empire byzantin (Gülgönen \& Bilsel, 1991 cités par Cankat, 2011: 87 - la référence précédente à Yerasimos est aussi tirée de Cankat).

3. Si ce discours participe à la légitimation de classes montantes, cela n'implique toutefois pas pour autant que la mise en valeur d'une identité conçue comme musulmane serait induite par l'embourgeoisement de nouveaux citadins d'origine anatolienne, au sens d'une diffusion d'un habitus spécifique, voire ontologique. Ce discours historique s'appuie sur une narrativité qui s'affirme dès les années 1970 et trouve son origine bien avant (Copeau, 1998).

4. Je reprends ici le qualificatif de « vernaculaire ", non dans le sens où il est utilisé par l'ICOMOS et l'UNESCO ou appliqué aux maisons en bois de Zeyrek et Süleymaniye (où il remplace de fait 
«traditionnel »), mais pour rappeler ce que, via l'étymologie, ce terme a d'implicite (cf. Alain Rey, 2006. Dictionnaire historique de la langue française, Robert).

5. La plaque commémorative présente dans son mausolée, traduite en anglais, en précise la généalogie, les faits d'armes et les témoignages de sa foi. Faits sujets à caution puisque Selim I n'était alors pas calife. Contrairement à la thèse répandue d'un califat ottoman débutant avec la conquête de l'Egypte, la revendication de ce titre apparaît beaucoup plus tardivement (au XVIII siècle) dans les relations diplomatiques avec les pays européens (Sourdel, 1978 : 978).

6. La compilation et la reproduction d'éléments faisant référence au passé fournit en effet un ancrage et un véhicule historique - l'arche - qui fait autorité sur le présent - arkhein : commander, administrer. (Rey, 2006 ; Derrida, 1995)

7. Rémi Stoquart, décembre 1997. Projet de réhabilitation dues quartiers de Balat et de Fener, diagnostic et premières orientations opérationnelles. Rapport intermédiaire.

8. Selon les termes d'une Américaine venue s'installer à Fener il y a environ 5 ans (entretien exploratoire mené en mai 2010).

9. Représentant élu du mahalle, juridiquement non-affilié à quelconque parti politique

10. Parti formé suite à la dissolution du Refah, par le fondateur de l'islam politique en Turquie, l'ex-Premier ministre Erbakan (dont les funérailles nationales ont eu lieu le $1^{\mathrm{er}}$ mars 2011 à la grande mosquée de Fatih). Le Saadet se place dans l'opposition à l'AKP, sur une ligne contestant notamment ses orientations néo-libérales.

11. Selon les propres termes de l'association.

12. Bien que le Patriarcat soit héritier d'une institution byzantine, son installation en ces lieux date de l'Empire ottoman et son statut actuel de la République turque. Cela peut donc sembler paradoxal que le lien entre le Patriarcat et l'héritage byzantin transite par une référence qui tend à rapprocher l'identité rum de l'identité grecque. L'idée nationale grecque s'est en effet construite contre l'Empire et entretient donc des rapports ambigus avec l'identité rum, a fortiori à Istanbul ; la catégorie Rum ne peut précisément subsister que dans sa relation à l'Empire ottoman (qui lui donne son statut juridique) et la république turque. Pour contrebalancer l'idée que les rum seraient grecs, rappelons par exemple que la majorité des élèves du lycée rum de Fener sont arabophones.

13. Selon les termes du muhtar, interrogé sur les problèmes du quartier (février 2010).

14. Chiffres de février 2011.

15. Image derrière laquelle se développent des pratiques et des standards tout à fait contemporains : outre ses fonctions religieuses, « jet Hoca » est aussi connu pour son goût pour le luxe. Par exemple, le 22 mars 2011 il bénissait à Bayrampaşa l'inauguration du Caprice Gold Otel, un établissement « 7 étoiles » parfaitement hallal.

16. Notons que l'orthodoxie affichée est beaucoup plus souple quand il s'agit de grandir les titres d'un personnage qui ni s'est réclamé calife, ni semblait en mesure de la faire au regard de la Loi Coranique (Sourdel, 1978)

17. «Istanbul'un en güvenli ve Huzurlu mahallesi, Balat », in Fatih, bulletin municipal édité par la mairie d'arrondissement de Fatih, n³7, février 2011, p 7.

18. Entendu en référence aux familles kurdes, accusées de cracher dans la rue et de boire (les souillures se renforçant mutuellement). 


\section{RÉSUMÉS}

La mise en patrimoine de la péninsule historique d'Istanbul nourrit un processus complexe de changement urbain. Participant à la redéfinition des fonctions économiques et au renouvellement, parfois très brutal, de l'habitat de la presqu'île, elle engendre des polémiques qui rejoignent les débats politiques voire idéologiques portant sur des questions sociétales centrales. Les discours portés sur cet espace à forte charge symbolique interrogent ainsi directement ce qui fait ou devrait faire lien social, la ville et ses quartier apparaissant souvent comme une métonymie de la société turque dans son ensemble. Partant d'observations menées dans deux quartiers voisins, l'un (Fener) connu et fréquenté pour son patrimoine rum, l'autre (Çarşamba) cultivant une image islamique, j'en questionne les frontières symboliques, les relations de distances et de proximités qui s'y développent.

The transformation and preservation of Istanbul's historic peninsula into protected historical heritage underpins a complex process of urban change. Contributing to the redefinition of the economic functions and the sometimes brutal renovation of the living space of the almost-island, the process engenders polemics that catch-up with the current political - even ideological debates about fundamental societal questions. The discourses about this strongly symbolic space scrutinise directly what is (or should be) the social link; the city and its neighbourhoods are often seen as a metonymy of the entire Turkish society. With the help of observations conducted in two neighbourhoods - one (Fener) known for and visited because of its heritage rum, the other one (Çarşamba) cultivating an Islamic image - I explore the relations of distance and proximity and the symbolic borders that are both developing in these two districts.

\section{INDEX}

Keywords : Çarşamba, Fatih, Fener, heritage, Istanbul, ottomanism, Turkey

Mots-clés : Çarşamba, Fatih, Fener, Istanbul, ottomanisme, patrimoine, Turquie

Thèmes : Sur le Champ - Sur le Terrain

\section{AUTEUR}

\section{BRIAN CHAUVEL}

Brian Chauvel est doctorant en sociologie EHESS-Paris, boursier Tübitak. Sa thèse, sous la direction de Nilüfer Göle, porte sur "Enjeux patrimoniaux et renouvellement urbain dans la péninsule historique d'Istanbul". brian.chauvel@gmail.com 